\title{
Multidimensional Harmonic Retrieval via Coupled Canonical Polyadic Decomposition Part I: Model and Identifiability
}

\author{
Mikael Sørensen and Lieven De Lathauwer, Fellow, IEEE
}

\begin{abstract}
Multidimensional Harmonic Retrieval (MHR) is a fundamental problem in signal processing. We make a connection with coupled Canonical Polyadic Decomposition (CPD), which allows us to better exploit the rich MHR structure than existing approaches in the derivation of uniqueness results. We discuss both deterministic and generic conditions. We obtain a deterministic condition that is both necessary and sufficient but which may be difficult to check in practice. We derive mild deterministic relaxations that are easy to verify. We also discuss the variant in which the generators have unit norm. We narrow the transition zone between generic uniqueness and generic non-uniqueness to two values of the number of harmonics. We explain differences with one-dimensional HR.
\end{abstract}

Index Terms-coupled canonical polyadic decomposition, tensor, Vandermonde matrix, multidimensional harmonic retrieval.

\section{INTRODUCTION}

During the past two decades Multidimensional Harmonic Retrieval (MHR) has become an important problem in signal processing. MHR is a fundamental problem that appears in a wide range of applications in traditional signal processing, such as radar, sonar, wireless communication and channel sounding, see [32], [16], [19], [24], [22], [23], [13], [37] and references therein. The MHR structure can be due to Doppler effects, structured receive and/or transmit antenna arrays, sinusoidal carriers, carrier frequency off-sets, and so on. Another classical signal processing application of MHR is multidimensional NMR spectroscopy (e.g. [21]). More recent MHR applications in signal processing include sampling of parametric nonbandlimited 2D signals [25], phase retrieval of parametric 2D signals [31], phase retrieval

M. Sørensen and L. De Lathauwer are with KU Leuven - E.E. Dept. (ESAT) - STADIUS Center for Dynamical Systems, Signal Processing and Data Analytics, Kasteelpark Arenberg 10, B-3001 Leuven-Heverlee, Belgium, the Group Science, Engineering and Technology, KU Leuven Kulak, E. Sabbelaan 53, 8500 Kortrijk, Belgium, and iMinds Medical IT, Kasteelpark Arenberg 10, B-3001 Leuven-Heverlee, Belgium, \{Mikael.Sorensen, Lieven.DeLathauwer\}@kuleuven.be.

Research supported by: (1) Research Council KU Leuven: $\mathrm{CoE} \mathrm{EF} / 05 / 006$ Optimization in Engineering (OPTEC), C1 project C16/15/059-nD, (2) F.W.O.: project G.0830.14N, G.0881.14N, (3) the Belgian Federal Science Policy Office: IUAP P7 (DYSCO II, Dynamical systems, control and optimization, 2012-2017), (4) EU: The research leading to these results has received funding from the European Research Council under the European Union's Seventh Framework Programme (FP7/2007-2013) / ERC Advanced Grant: BIOTENSORS (no. 339804). This paper reflects only the authors' views and the Union is not liable for any use that may be made of the contained information. of multidimensional spike models [2] and antenna array design [26]. Thus, a better understanding of MHR will also lead to more insights in a wide range of problems in signal processing. There is also an increasing interest in multiway signal processing (e.g., [4]), which further motivates the study of multidimensional extensions of classical problems, such as one-dimensional (1D) Harmonic Retrieval (HR).

A link between MHR and the Canonical Polyadic Decomposition (CPD) was established in [32]. Roughly speaking, it was observed that MHR problems can be addressed as Vandermonde constrained CPD (VDM$\mathrm{CPD})$ problems. Based on the VDM-CPD, uniqueness conditions and algebraic methods for MHR have been developed in [32], [16], [24], [22], [23]. In fact, such VDMCPD approaches do not fully exploit the structure of the MHR problem. More precisely, after a data preprocessing step, the existing results in fact infer conclusions for the MHR problem from one of the individual harmonic structures. Hence, a tool that can simultaneously exploit several harmonic structures is of interest.

The authors have recently extended the CPD modeling framework to coupled models in [38], [41]. Coupled matrix/tensor decompositions are basic tools for data fusion, i.e., for the joint analysis of multiple related data sets. Data fusion has important applications in telecommunication, biomedical signal processing, chemometrics, bioinformatics, social network analysis, artificial intelligence, etc., see [38], [41], [35], [34] and references therein.

In this paper, we present a link between MHR and the coupled CPD modeling framework [38], [41]. Briefly, as in classical ESPRIT [29], each harmonic structure of the given MHR problem can be associated with a low-rank CPD structure [32]. An interesting property of the coupled CPD approach is that it allows one to take several of the harmonic structures into account at once. For this reason, coupled CPD leads to improved uniqueness conditions. As we will explain, in some cases we even obtain conditions that are both necessary and sufficient and hence fully exploit the MHR structure. In the companion paper [39], we discuss algorithmic aspects and explain that the coupled CPD supports multirate sampling. Part of this work appeared in the conference paper [36].

Despite the conceivable use of coupled tensor decompositions for source separation, data compression 
and other related signal processing applications, coupled CPD (and it variants) has so far received little attention in the signal processing community. Thus, another goal of this paper is to raise the awareness of the potential use of coupled tensor decompositions. We use the timeless and ubiquitous MHR problem as an illustrative example. In the same way as the discovery of the link between CPD and sensor array processing in [33] sparked the interest in CPD-based signal processing, we expect that the coupled CPD will find many relevant applications in signal processing engineering.

The paper is organized as follows. The rest of the introduction will present the notation used throughout the paper. Section II reviews the necessary algebraic prerequisites. In Section IIII we briefly discuss the connection between 1D HR and CPD. Section IV presents new uniqueness conditions for MHR. In particular, a link between MHR and coupled CPD will be introduced that in some cases fully exploit the MHR structure. Next, in Section $\mathrm{V}$ we discuss and compare the outcomes of Section IV with existing MHR identifiability results. Section VI concludes the paper.

Notation: Vectors, matrices and tensors are denoted by lower case boldface, upper case boldface and upper case calligraphic letters, respectively. The modulus of $a \in \mathbb{C}$ is denoted by $|a|$. The $r$ th column vector of $\mathbf{A}$ is denoted by $\mathbf{a}_{r}$. The symbols $\otimes$ and $\odot$ denote the Kronecker and Khatri-Rao product, defined as

$$
\begin{aligned}
& \mathbf{A} \otimes \mathbf{B}:=\left[\begin{array}{ccc}
a_{11} \mathbf{B} & a_{12} \mathbf{B} & \ldots \\
a_{21} \mathbf{B} & a_{22} \mathbf{B} & \ldots \\
\vdots & \vdots & \ddots
\end{array}\right], \\
& \mathbf{A} \odot \mathbf{B}:=\left[\mathbf{a}_{1} \otimes \mathbf{b}_{1} \mathbf{a}_{2} \otimes \mathbf{b}_{2} \ldots\right],
\end{aligned}
$$

in which $(\mathbf{A})_{m n}=a_{m n}$. We denote the Kronecker and Khatri-Rao products of $N$ matrices $\left\{\mathbf{A}^{(n)}\right\}_{n=1}^{N}$ by $\bigotimes_{n=1}^{N} \mathbf{A}^{(n)}=\mathbf{A}^{(1)} \otimes \cdots \otimes \mathbf{A}^{(N)}$ and $\bigodot_{n=1}^{N} \mathbf{A}^{(n)}=\mathbf{A}^{(1)} \odot$ $\cdots \odot \mathbf{A}^{(N)}$, respectively. The symbol $\mathbf{A} * \mathbf{B}$ denotes the Hadamard product, i.e., $(\mathbf{A} * \mathbf{B})_{i j}=a_{i j} b_{i j}$ and $*_{n=1}^{N} \mathbf{A}^{(n)}=$ $\mathbf{A}^{(1)} * \mathbf{A}^{(2)} * \cdots * \mathbf{A}^{(N)}$. The Cartesian product of $N$ sets $\left\{\mathbb{C}^{I_{n}}\right\}_{n=1}^{N}$ is denoted by $\mathbb{C}^{\stackrel{N}{N} I_{n}}=\mathbb{C}^{I_{1} \times \cdots \times I_{N}}$. The outer product of $N$ vectors $\mathbf{a}^{(n)} \in \mathbb{C}^{I_{n}}$ is denoted by $\mathbf{a}^{(1)} \otimes \cdots \otimes$ $\mathbf{a}^{(N)} \in \mathbb{C}^{I_{1} \times I_{2} \times \cdots \times I_{N}}$, such that $\left(\mathbf{a}^{(1)} \otimes \cdots \otimes \mathbf{a}^{(N)}\right)_{i_{1}, i_{2}, \ldots, i_{N}}=$ $a_{i_{1}}^{(1)} a_{i_{2}}^{(2)} \cdots a_{i_{N}}^{(N)}$.

The conjugate, transpose, conjugate-transpose and Moore-Penrose pseudo-inverse of the matrix $\mathbf{A}$ are denoted by $\mathbf{A}^{*}, \mathbf{A}^{T}, \mathbf{A}^{H}$ and $\mathbf{A}^{\dagger}$, respectively. The number of nonzero entries of a vector $\mathbf{a}$ is denoted by $\|\mathbf{a}\|_{0}$. From the context it should be clear when $i$ denotes the imaginary unit number, i.e., $i=\sqrt{-1}$.

The identity matrix and all-zero vector are denoted by $\mathbf{I}_{R} \in \mathbb{C}^{R \times R}$ and $\mathbf{0}_{R} \in \mathbb{C}^{R}$, respectively. The exchange matrix with unit entries on the antidiagonal and zeros elsewhere is denoted by $\mathbf{J}_{I} \in \mathbb{C}^{I \times I}$.

Matlab index notation will be used for submatrices of a given matrix. For example, $\mathbf{A}(1: k,:)$ represents the submatrix of $\mathbf{A}$ consisting of the rows from 1 to $k$ of $\mathbf{A}$.
Let $\mathbf{A} \in \mathbb{C}^{I \times J}$, then $\underline{\mathbf{A}}=\mathbf{A}(1: I-1,:) \in \mathbb{C}^{(I-1) \times J}$ and $\overline{\mathbf{A}}=\mathbf{A}(2: I,:) \in \mathbb{C}^{(\bar{I}-1) \times J}$, i.e., $\underline{\mathbf{A}}$ and $\overline{\mathbf{A}}$ are obtained by deleting the bottom and top row of $\mathbf{A}$, respectively. The vectorization of the matrix $\mathbf{A} \in \mathbb{C}^{I \times J}$ is denoted by $\operatorname{Vec}(\mathbf{A})=\left[\mathbf{a}_{1}^{T}, \mathbf{a}_{2}^{T}, \ldots, \mathbf{a}_{J}^{T}\right]^{T} \in \mathbb{C}^{I J} . D_{k}(\mathbf{A}) \in \mathbb{C}^{J \times J}$ denotes the diagonal matrix holding row $k$ of $\mathbf{A} \in \mathbb{C}^{I \times J}$ on its diagonal.

The $k$-th compound matrix of $\mathbf{A} \in \mathbb{C}^{I \times R}$ is denoted

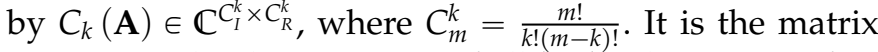
containing the determinants of all $k \times k$ submatrices of $\mathbf{A}$, arranged with the submatrix index sets in lexicographic order. See [14], [7] and references therein for a discussion of compound matrices.

The $k$-rank of a matrix $\mathbf{A}$ is denoted by $k_{\mathbf{A}}$. It is equal to the largest integer $k_{\mathrm{A}}$ such that every subset of $k_{\mathrm{A}}$ columns of $\mathbf{A}$ is linearly independent.

\section{Algebraic Foundations}

\section{A. Canonical Polyadic Decomposition (CPD)}

Consider the third-order tensor $\mathcal{X} \in \mathbb{C}^{I \times J \times K}$. We say that $X$ is a rank- 1 tensor if it is equal to the outer product of some non-zero vectors $\mathbf{a} \in \mathbb{C}^{I}, \mathbf{b} \in \mathbb{C}^{J}$ and $\mathbf{c} \in \mathbb{C}^{K}$ such that $x_{i j k}=a_{i} b_{j} c_{k}$. A Polyadic Decomposition (PD) is a decomposition of $\mathcal{X}$ into rank-1 terms:

$$
\mathbb{C}^{I \times J \times K} \ni \mathcal{X}=\sum_{r=1}^{R} \mathbf{a}_{r} \otimes \mathbf{b}_{r} \otimes \mathbf{c}_{r} .
$$

The rank of a tensor $X$ is equal to the minimal number of rank-1 tensors that yield $\mathcal{X}$ in a linear combination. Assume that the rank of $\mathcal{X}$ is $R$, then (1) is called the CPD of $\mathcal{X}$, i.e., a PD of $\mathcal{X}$ with a minimal number of terms is a CPD. Let us stack the vectors $\left\{\mathbf{a}_{r}\right\},\left\{\mathbf{b}_{r}\right\}$ and $\left\{\mathbf{c}_{r}\right\}$ into the matrices $\mathbf{A}=\left[\mathbf{a}_{1}, \ldots, \mathbf{a}_{R}\right], \mathbf{B}=\left[\mathbf{b}_{1}, \ldots, \mathbf{b}_{R}\right]$ and $\mathbf{C}=\left[\mathbf{c}_{1}, \ldots, \mathbf{c}_{R}\right]$. The matrices $\mathbf{A}, \mathbf{B}$ and $\mathbf{C}$ will be referred to as the factor matrices of the PD or CPD of $\mathcal{X}$ in (1).

1) Matrix Representations: Consider the horizontal matrix slice $\boldsymbol{X}^{(i \cdot k)} \in \mathbb{C}^{J \times K}$ of $\mathcal{X}$, defined by $\left(\mathbf{X}^{(i \cdot .)}\right)_{j k}=$ $x_{i j k}=\sum_{r=1}^{R} a_{i r} b_{j r} c_{k r}$. The tensor $\mathcal{X}$ can be interpreted as a collection of matrices $\mathbf{X}^{(1 \cdot \cdot)}, \ldots, \mathbf{X}^{(I \cdot)}$, yielding the slicewise representation of (1):

$$
\mathbf{X}^{(i . .)}=\sum_{r=1}^{R} a_{i r} \mathbf{b}_{r} \mathbf{c}_{r}^{T}=\mathbf{B} D_{i}(\mathbf{A}) \mathbf{C}^{T} .
$$

Stacking yields the classical matrix representation (e.g., [17], [18], [4]):

$$
\mathbb{C}^{I J \times K} \ni \mathbf{X}:=\left[\begin{array}{c}
\mathbf{X}^{(1 \cdot \cdot)} \\
\vdots \\
\mathbf{X}^{(I \cdot \cdot)}
\end{array}\right]=\left[\begin{array}{c}
\mathbf{B} D_{1}(\mathbf{A}) \\
\vdots \\
\mathbf{B} D_{I}(\mathbf{A})
\end{array}\right] \mathbf{C}^{T}=(\mathbf{A} \odot \mathbf{B}) \mathbf{C}^{T} .
$$


2) Uniqueness: The rank-1 tensors in (1) can be arbitrarily permuted without changing the decomposition. The vectors within the same rank-1 tensor can also be arbitrarily scaled provided that the overall rank-1 term remains the same. The CPD is said to be unique when it is only subject to the mentioned indeterminacies. The development of uniqueness conditions for the CPD has been the subject of intensive investigation, see [7], [8] and references therein. In this paper we assume that $\mathbf{C}$ in (3) has full column rank (possibly after spatial smoothing and/or FBA, see Section II-C).

For the case where $\mathbf{C}$ has full column rank, it was observed in [44], [15] that a necessary and sufficient condition for CPD uniqueness is that no linear combination of the columns of $\mathbf{A} \odot \mathbf{B}$ can be written as a vectorized rank-1 matrix. This can be expressed more formally as

$$
\sum_{r=1}^{R} \mathbf{a}_{r} \mathbf{b}_{r}^{T} d_{r}=\mathbf{e f}^{T} \Rightarrow\|\mathbf{d}\|_{0} \leqslant 1,
$$

for some e $\in \mathbb{C}^{I}, \mathbf{f} \in \mathbb{C}^{J}$ and $\mathbf{d}=\left[d_{1}, \ldots, d_{R}\right]^{T} \in \mathbb{C}^{R}$. In terms of compound matrices, the necessary and sufficient condition (4) can be formulated as follows.

Theorem II.1. Consider the PD of $\mathcal{X} \in \mathbb{C}^{I \times J \times K}$ in (1). Assume that $C$ has full column rank. The rank of $\mathcal{X}$ is $R$ and the CPD of $\mathcal{X}$ is unique if and only if [15], [7]:

$$
\left(C_{2}(A) \odot C_{2}(B)\right) \boldsymbol{d}^{(2)}=\mathbf{0} \Rightarrow\|\boldsymbol{d}\|_{0} \leqslant 1 ，
$$

where $\boldsymbol{d}^{(2)}=\left[d_{1} d_{2}, d_{1} d_{3}, \ldots, d_{R-1} d_{R}\right]^{T} \in \mathbb{C}^{C_{R}^{2}}$. Generically 1$]$ condition (5) is satisfied if and only if $R \leqslant(I-1)(J-1)$ [44], [3], [10].

The necessary and sufficient condition (5) can be hard to check in practice. Observe that if $C_{2}(\mathbf{A}) \odot C_{2}(\mathbf{B})$ in (5) has full column rank, then $\mathbf{d}^{(2)}=\mathbf{0}$ and the condition is immediately satisfied. This fact leads to the following easy-to-check uniqueness condition, which is only sufficient.

Theorem II.2. Consider the PD of $\mathcal{X} \in \mathbb{C}^{I \times J \times K}$ in (1). If

$$
\left\{\begin{array}{l}
\boldsymbol{C} \text { has full column rank, } \\
C_{2}(A) \odot C_{2}(B) \text { has full column rank, }
\end{array}\right.
$$

then the rank of $\mathcal{X}$ is $R$ and the CPD of $\mathcal{X}$ is unique [15], [6], [7]. Generically, conditions (6a) and (6b) are satisfied if $R \leqslant K$ and $2 R(R-1) \leqslant I(I-1) J(J-1)[6]$, [43].

Tightening the condition in Theorem III.1 to that in Theorem II.2 has the additional advantage that the CPD can be computed under conditions (6a) and $6 \mathrm{~b}$ by means of linear algebra (low-rank matrix approximation and EigenValue Decomposition (EVD)); the robustness of the computation can be increased via Simultaneous matrix Diagonalization (SD) techniques [6], [9].

\footnotetext{
${ }^{1} \mathrm{~A}$ generic property is a property that holds everywhere except for a set of Lebesgue measure zero. In particular, a generic tensor decomposition property is a property that holds with probability one if the entries of the factor matrices are randomly drawn from continuous distributions.
}

In the case where two factor matrices, say $\mathbf{A}$ and $\mathbf{C}$, have full column rank, Theorem II.2 simplifies to the following. (It can be verified that if $\mathbf{A}$ has full column rank and $k_{\mathbf{B}} \geqslant 2$, then $C_{2}(\mathbf{A}) \odot C_{2}(\mathbf{B})$ also has full column rank.)

Theorem II.3. Consider the PD of $\mathcal{X}$ in (1). Assume that $A$ and $C$ have full column rank. The rank of $\mathcal{X}$ is $R$ and the $C P D$ of $X$ is unique if and only if $k_{B} \geqslant 2$ (e.g. [20]). Generically, this is satisfied if $R \leqslant \min (I, K)$ and $2 \leqslant J$.

Furthermore, if the conditions stated in Theorem II.3 are satisfied, then the CPD of $\mathcal{X}$ follows directly from an EVD (e.g. [20]). As a matter of fact, in the constructive proof of Theorem III.2 the computation of the CPD is reduced to a computation under the conditions in Theorem II.3. This will be further discussed in the companion paper [39].

\section{B. Coupled CPD}

We say that a collection of tensors $\mathcal{X}^{(n)} \in \mathbb{C}^{I_{n} \times J_{n} \times K}, n \in$ $\{1, \ldots, N\}$, admits an $R$-term coupled PD if each tensor $\mathcal{X}^{(n)}$ can be written as [38]:

$$
\boldsymbol{X}^{(n)}=\sum_{r=1}^{R} \mathbf{a}_{r}^{(n)} \otimes \mathbf{b}_{r}^{(n)} \otimes \mathbf{c}_{r}, \quad n \in\{1, \ldots, N\},
$$

with factor matrices $\mathbf{A}^{(n)}=\left[\mathbf{a}_{1}^{(n)}, \ldots, \mathbf{a}_{R}^{(n)}\right], \mathbf{B}^{(n)}=$ $\left[\mathbf{b}_{1}^{(n)}, \ldots, \mathbf{b}_{R}^{(n)}\right]$ and $\mathbf{C}=\left[\mathbf{c}_{1}, \ldots, \mathbf{c}_{R}\right]$. We define the coupled rank of $\left\{\boldsymbol{X}^{(n)}\right\}$ as the minimal number of coupled rank-1 tensors $\mathbf{a}_{r}^{(n)} \otimes \mathbf{b}_{r}^{(n)} \otimes \mathbf{c}_{r}$ that yield $\left\{\boldsymbol{X}^{(n)}\right\}$ in a linear combination. Assume that the coupled rank of $\left\{\mathcal{X}^{(n)}\right\}$ is $R$, then (7) will be called the coupled CPD of $\left\{\mathcal{X}^{(n)}\right\}$.

1) Matrix representation: The coupled (C)PD of $\left\{\mathcal{X}^{(n)}\right\}$ given by (7) has the following matrix representation

$\mathbf{X}=\left[\begin{array}{c}\mathbf{X}^{(1)} \\ \vdots \\ \mathbf{X}^{(N)}\end{array}\right]=\left[\begin{array}{c}\mathbf{A}^{(1)} \odot \mathbf{B}^{(1)} \\ \vdots \\ \mathbf{A}^{(N)} \odot \mathbf{B}^{(N)}\end{array}\right] \mathbf{C}^{T} \in \mathbb{C}^{\left(\sum_{n=1}^{N} I_{n} J_{n}\right) \times K}$.

2) Uniqueness: The coupled rank-1 tensors in (7) can be arbitrarily permuted and the vectors within the same coupled rank-1 tensor can be arbitrarily scaled provided the overall coupled rank-1 term remains the same. We say that the coupled CPD is unique when it is only subject to these trivial indeterminacies. Uniqueness conditions for the coupled CPD were derived in [38]. For the case where $\mathbf{C}$ has full column rank, Theorem II.4 below is an extension of Theorem II.1 to the coupled CPD case. It will make use of the matrix

$$
\mathbf{G}=\left[\begin{array}{c}
C_{2}\left(\mathbf{A}^{(1)}\right) \odot C_{2}\left(\mathbf{B}^{(1)}\right) \\
\vdots \\
C_{2}\left(\mathbf{A}^{(N)}\right) \odot C_{2}\left(\mathbf{B}^{(N)}\right)
\end{array}\right] \in \mathbb{C}^{\left(\sum_{n=1}^{N} C_{I n}^{2} C_{I n}^{2}\right) \times C_{R}^{2}}
$$

Theorem II.4. Consider the coupled PD of $\mathcal{X}^{(n)} \in \mathbb{C}^{I_{n} \times J_{n} \times K}$, $n \in\{1, \ldots, N\}$ in (7). Assume that $C$ has full column rank. 
The coupled rank of $\left\{\mathcal{X}^{(n)}\right\}$ is $R$ and the coupled CPD of $\left\{X^{(n)}\right\}$ is unique if and only if

$$
\boldsymbol{G} \boldsymbol{d}^{(2)}=\mathbf{0} \Rightarrow\|\boldsymbol{d}\|_{0} \leqslant 1,
$$

where $\boldsymbol{d}^{(2)}=\left[d_{1} d_{2}, d_{1} d_{2}, \ldots, d_{R-1} d_{R}\right]^{T} \in \mathbb{C}_{R}^{C_{R}^{2}}[38]$.

As in the ordinary CPD case, the necessary and sufficient uniqueness condition (10) can be hard to check in practice. Theorem III.5 extends the easy-to-check condition in Theorem II.2 to coupled CPD.

Theorem II.5. Consider the coupled PD of $\mathcal{X}^{(n)} \in \mathbb{C}^{I_{n} \times J_{n} \times K}$, $n \in\{1, \ldots, N\}$ in (7). If

$$
\left\{\begin{array}{l}
C \text { in (8) has full column rank, } \\
G \text { in (9) has full column rank, }
\end{array}\right.
$$

then the coupled rank of $\left\{X^{(n)}\right\}$ is $R$ and the coupled CPD of $\left\{\mathcal{X}^{(n)}\right\}$ is unique 38].

Comparing Theorem II.2 with Theorem II.5, we observe that the coupling in (7) leads to a more relaxed uniqueness condition. As in ordinary CPD, under conditions (11a) and (11b) the coupled CPD can also be computed via a GEVD. This will be discussed in detail in the companion paper [39].

In case at least one of the other factor matrices has full column rank as well, we may use the following Theorem II.6, which can be understood as an extension of Theorem III.3 to coupled CPD.

Theorem II.6. Consider the coupled PD of $\left\{\boldsymbol{X}^{(n)}\right\}$ in (7). If there exists a subset $S \subseteq\{1, \ldots, N\}$ such that

$\left\{\begin{array}{l}C \text { has full column rank, } \\ A^{(n)} \text { has full column rank, } \forall n \in S, \\ \forall r \in\{1, \ldots, R\}, \forall s \in\{1, \ldots, R\} \backslash r, \exists n \in S: k_{\left[b_{r}^{(n)}, b_{s}^{(n)}\right]}=2,\end{array}\right.$

then the coupled rank of $\left\{\mathcal{X}^{(n)}\right\}$ is $R$ and the coupled CPD of $\left\{\boldsymbol{X}^{(n)}\right\}$ is unique 38].

Note that the cardinality of the chosen subset $S$ allows one to trade off full column rank in the first mode for non-collinearity in the second mode.

\section{Vandermonde matrices}

The matrix $\mathbf{A} \in \mathbb{C}^{I \times R}$ is called Vandermonde if

$$
\mathbf{A}=\left[\mathbf{a}_{1}, \ldots, \mathbf{a}_{R}\right], \quad \mathbf{a}_{r}=\left[1, z_{r}, z_{r}^{2}, \ldots, z_{r}^{I-1}\right]^{T},
$$

where the scalars $\left\{z_{r}\right\}$ are called the generators of $\mathbf{A}$.

1) Shift-invariance: The key attribute of Vandermonde matrices in the context of MHR is the shift-invariance property $\overline{\mathbf{a}}_{r}=\underline{\mathbf{a}}_{r} \cdot z_{r}$. This property can be translated into a rank-1 structure. Indeed, each column of

$$
\left[\begin{array}{l}
\overline{\mathbf{A}}
\end{array}\right]=\left[\begin{array}{lll}
\underline{\mathbf{a}}_{1} & \cdots & \underline{\mathbf{a}}_{R} \\
\underline{\mathbf{a}}_{1} z_{1} & \cdots & \underline{\mathbf{a}}_{R} z_{R}
\end{array}\right]=\left[\begin{array}{l}
\underline{\mathbf{A}} \\
\underline{\mathbf{A}} D_{2}(\mathbf{A})
\end{array}\right]=\mathbf{A}^{(2)} \odot \underline{\mathbf{A}}
$$

is a vectorized rank-1 $(2 \times(I-1))$ Hankel matrix, where $\mathbf{A}^{(2)}=\left[\begin{array}{ccc}1 & \cdots & 1 \\ z_{1} & \cdots & z_{R}\end{array}\right] \in \mathbb{C}^{2 \times R}$. The relation between Vandermonde and more general rank-1 matrices leads to the concept of spatial smoothing.
2) Spatial smoothing: Using the property $z^{l+k-2}=z^{l-1} z^{k-1}$, the Vandermonde vector $\mathbf{a}=\left[1, z, z^{2}, \ldots, z^{I-1}\right]^{T} \in \mathbb{C}^{I}$ can be mapped to the rank-1 $(L \times K)$ Hankel matrix $\mathbf{Y}$ :

$$
\mathbf{Y}=\left[\begin{array}{cccc}
a_{1} & a_{2} & \cdots & a_{K} \\
a_{2} & a_{3} & & \vdots \\
\vdots & & a_{I-2} & a_{I-1} \\
a_{L} & \cdots & a_{I-1} & a_{I}
\end{array}\right]=\mathbf{a}^{(L)} \mathbf{a}^{(K) T},
$$

where $K+L=I+1$ and where $\mathbf{a}^{(K)}=\left[1 z z^{2} \ldots z^{K-1}\right]^{T} \in$ $\mathbb{C}^{K}$ and $\mathbf{a}^{(L)}=\left[\begin{array}{lllll}1 & z & z^{2} & \ldots & z^{L-1}\end{array}\right]^{T} \in \mathbb{C}^{L}$. The vectorized version of $\mathbf{Y}$ admits the factorization $\mathbf{y}=\operatorname{Vec}(\mathbf{Y})=\mathbf{a}^{(K)} \otimes$ $\mathbf{a}^{(L)}$. This "splitting" of a Vandermonde vector has been used in the context of signal processing since the eighties and is sometimes called spatial smoothing (e.g., [30]). The following paragraphs discuss two applications of spatial smoothing that will be used in the paper.

a) Spatial smoothing to increase the system diversity: Consider the factorization $\mathbf{X}=\mathbf{A C}^{T} \in \mathbb{C}^{I \times M}$ in which $\mathbf{A} \in \mathbb{C}^{I \times R}$ is a Vandermonde matrix and $\mathbf{C} \in \mathbb{C}^{M \times R}$. Using $z_{r}^{l+k-2}=z_{r}^{l-1} z_{r}^{k-1}$, spatial smoothing maps the matrix $\mathbf{X}$ to the tensor $\boldsymbol{Y} \in \mathbb{C}^{L \times K \times M}$ :

$$
y_{l, k, m}=x_{k+l-1, m}=\sum_{r=1}^{R} a_{k+l-1, r} c_{m, r}=\sum_{r=1}^{R} z_{r}^{l-1} z_{r}^{k-1} c_{m, r},
$$

where $1 \leqslant k \leqslant K$ and $1 \leqslant l \leqslant L$ are subject to $K+L=I+1$. Consider now the frontal matrix slices of $\mathcal{Y}$, defined by $\mathbf{Y}_{1}=\mathcal{Y}(:,:, 1) \in \mathbb{C}^{L \times K}, \ldots, \mathbf{Y}_{M}=\mathcal{Y}(:,:, M) \in \mathbb{C}^{L \times K}$. Comparing (14) and (15) for fixed $m \in\{1, \ldots, M\}$, it becomes clear that $y$ is a collection of stacked Hankel matrices, each with factorization $\mathbf{Y}_{m}=\sum_{r=1}^{R} \mathbf{a}_{r}^{(L)} \mathbf{a}_{r}^{(K) T} c_{m r}$, where $\mathbf{a}_{r}^{(K)}=\left[\begin{array}{llllll}1 & z_{r} & z_{r}^{2} & \ldots & z_{r}^{K-1}\end{array}\right]^{T}$ and $\mathbf{a}_{r}^{(L)}=\left[\begin{array}{lllll}1 & z_{r} & z_{r}^{2} & \ldots & z_{r}^{L-1}\end{array}\right]^{T}$. Hence, the "splitting" of the Vandermonde vectors in A leads to the PD (cf. (1)):

$$
\boldsymbol{y}=\sum_{r=1}^{R} \mathbf{a}_{r}^{(L)} \otimes \mathbf{a}_{r}^{(K)} \otimes \mathbf{c}_{r},
$$

with matrix representation (cf. 3)):

$$
\mathbf{Y}=\left[\operatorname{Vec}\left(\mathbf{Y}_{1}\right), \ldots, \operatorname{Vec}\left(\mathbf{Y}_{M}\right)\right]=\left(\mathbf{A}^{(K)} \odot \mathbf{A}^{(L)}\right) \mathbf{C}^{T},
$$

where $\mathbf{A}^{(K)}=\left[\begin{array}{lll}\mathbf{a}_{1}^{(K)} & \ldots & \mathbf{a}_{R}^{(K)}\end{array}\right] \in \mathbb{C}^{K \times R}$ and $\mathbf{A}^{(L)}=$ $\left[\begin{array}{lll}\mathbf{a}_{1}^{(L)} & \ldots & \mathbf{a}_{R}^{(L)}\end{array}\right] \in \mathbb{C}^{L \times R}$. Summarizing, the spatial smoothing has increased the order of the data array by one.

b) Spatial smoothing to obtain factor matrices that have full column rank: Consider again the factorization $\mathbf{X}=$ $\mathbf{A C}^{T} \in \mathbb{C}^{I \times M}$ in which $\mathbf{A} \in \mathbb{C}^{I \times R}$ is Vandermonde. We now focus on cases where $\mathbf{C} \in \mathbb{C}^{M \times R}$ does not have full column rank. By combining the second and third mode in (16), we obtain (cf. (17)):

$$
\mathbf{Z}=\mathbf{A}^{(K)}\left(\mathbf{A}^{(L)} \odot \mathbf{C}\right)^{T},
$$

where $\mathbf{Z}$ is built according to $z_{k,(l-1) L+m}=x_{k+l-1, m}$. In this way one may obtain factor matrices $\mathbf{A}^{(K)}$ and $\mathbf{A}^{(L)} \odot \mathbf{C}$ that both have full column rank. 
3) Forward-Backward Averaging (FBA): If the generators of the Vandermonde matrix $\mathbf{A}$ are located on the unit circle $\left(\left|z_{r}\right|=1\right)$, then we can also make use of the FBA procedure (e.g. [28]) to deal with rank deficiency. More precisely, consider the factorization $\mathbf{X}=\mathbf{A C ^ { T }}$ in which $\mathbf{A}$ is Vandermonde with generators $z_{r}=e^{i \alpha_{r}}$ where $\alpha_{r} \in \mathbb{R}$, $\forall r \in\{1, \ldots, R\}$. Then $\mathbf{J}_{I} \mathbf{A}^{*}=\mathbf{A D}_{\mathbf{A}}$ in which $\mathbf{D}_{\mathbf{A}}=$ $D_{1}\left(\left[z_{1}^{-(I-1)}, z_{2}^{-(I-1)}, \ldots, z_{R}^{-(I-1)}\right]\right)$. FBA now provides the augmented factorization $\left[\mathbf{X}, \mathbf{J}_{I} \mathbf{X}^{*}\right]=\mathbf{A}\left[\mathbf{C}^{T}, \mathbf{D}_{\mathbf{A}} \mathbf{C}^{H}\right]$. In short, FBA virtually doubles the amount of data samples and the augmented matrix $\left[\mathbf{C}^{T}, \mathbf{D}_{\mathbf{A}} \mathbf{C}^{H}\right]^{T}$ may have full column rank in cases where $\mathbf{C}$ has not. Generically, the rank of $\left[\mathbf{C}^{T}, \mathbf{D}_{\mathrm{A}} \mathbf{C}^{H}\right]^{T}$ is $\min (2 M, R)$ while the rank of $\mathbf{C}$ is only $\min (M, R)$, see for instance [40]. FBA has the advantage over spatial smoothing that the expansion of the matrix $\mathrm{C}$ is not compensated by a reduction of the matrix A. If FBA does not suffice to handle the rank deficiency, then it may be combined with spatial smoothing.

\section{Connections between 1D HR And CPD}

The 1D HR problem has implicitly been solved via CPD since the eighties [27], [29] and later on explicitly in [33]. In this section we will elaborate on the links between 1D HR and CPD. This will provide us with an understanding of why the coupled CPD approach introduced in section IV] is a natural framework for MHR.

Consider the 1D HR factorization

$$
\mathbf{X}=\mathbf{A} \mathbf{C}^{T} \in \mathbb{C}^{I \times M},
$$

where $\mathbf{A} \in \mathbb{C}^{I \times R}$ is a Vandermonde matrix and where $\mathbf{C} \in \mathbb{C}^{M \times R}$ is an unstructured matrix with full column rank. Note that since $\mathbf{C}$ has full column rank, the 1D HR factorization of $\mathbf{X}$ is unique if and only if the generators of $\mathbf{A}$ are distinct and $I>R$, which is equivalent to A having full column rank. The 'only if' part of this statement is obvious. Indeed, if $z_{r}=z_{s}$ for some $r \neq s$, then the decomposition (19) is not unique. Likewise, if $I \leqslant R$, then any Vandermonde matrix $\mathbf{V} \in \mathbb{C}^{I \times R}$ with $I$ distinct generators will yield an alternative factorization $\mathbf{X}=\mathbf{A} \mathbf{C}^{T}=\mathbf{V}\left(\mathbf{V}^{\dagger} \mathbf{A} \mathbf{C}^{T}\right)$. The 'if' part can be understood from the link with $C P D$, as will be discussed next.

\section{A. From $1 D$ HR to constrained CPD}

The shift-invariance property 13 of $\mathbf{A}$ implies that $\overline{\mathbf{X}}=\overline{\mathbf{A}} \mathbf{C}^{T}=\underline{\mathbf{A}} D_{2}(\mathbf{A}) \mathbf{C}^{T}$. Recall from Section II-A that $\underline{\mathbf{X}}=$ $\underline{\mathbf{A}} \mathbf{C}^{T}$ and $\overline{\mathbf{X}}=\underline{\mathbf{A}} D_{2}(\mathbf{A}) \mathbf{C}^{T}$ can be seen as slice-wise matrix representations of a PD, i.e., the 1D HR factorization (19) can be seen as a constrained CPD of $\mathcal{Y} \in \mathbb{C}^{2 \times(I-1) \times M}$ with matrix representation $\mathbf{Y} \in \mathbb{C}^{2(I-1) \times M}$ (cf. (3)):

$$
\left.\mathbf{Y}=\left[\begin{array}{l}
\mathbf{X} \\
\overline{\mathbf{X}}
\end{array}\right]=\left[\underline{\underline{\mathbf{A}}} \underline{\underline{\mathbf{A}} D_{2}(\mathbf{A})}\right]\right] \mathbf{C}^{T}=\left(\mathbf{A}^{(2)} \odot \mathbf{A}^{(I-1)}\right) \mathbf{C}^{T},
$$

where $\mathbf{A}^{(2)}=\left[\begin{array}{ccc}1 & \cdots & 1 \\ z_{1} & \cdots & z_{R}\end{array}\right] \in \mathbb{C}^{2 \times R}$ and $\mathbf{A}^{(I-1)}=\underline{\mathbf{A}} \in \mathbb{C}^{(I-1) \times R}$. As explained in Section II-C, (20) is a spatially smoothed variant of (19). In the notation of (17), we have $K=2$ and $L=I-1$, explaining the superscripts of $\mathbf{A}^{(2)}$ and $\mathbf{A}^{(I-1)}$. Note that, if we take the form of $\mathbf{A}^{(2)}$ and the Vandermonde structure of $\mathbf{A}^{(I-1)}$ into account, (19) and (20) are completely equivalent. In particular, the 1D HR factorization (19) of $\mathbf{X}$ is unique if and only if the constrained CPD (20) of $\boldsymbol{y}$ is unique. In the following paragraph we explain that the constraints can safely be ignored.

\section{B. From constrained $C P D$ to ordinary (unconstrained) $C P D$}

Due to the precise form of $\boldsymbol{Y}$ (which in turn exploits the shift-invariance), the Vandermonde constraints can be relaxed without affecting the uniqueness of the decomposition. Indeed, Theorem II.3 states that, if $k_{\mathbf{A}^{(2)}} \geqslant 2$ and the matrices $\mathbf{A}^{(I-1)}$ and $\mathbf{C}$ have full column rank, then CPD (20) is unique, even without imposing that $\mathbf{A}^{(2)}$ and $\mathbf{A}^{(I-1)}$ are Vandermonde. Note that this condition coincides with the 1D HR uniqueness condition, i.e., the Vandermonde generators are distinct if and only if $k_{\mathbf{A}^{(2)}} \geqslant 2$, and $\underline{\mathbf{A}}=\mathbf{A}^{(I-1)}$ has full column rank. To summarize, the 1D HR factorization of $\mathbf{X}$ is unique if and only if the unconstrained CPD of $\mathcal{Y}$ unique.

\section{CPD with a Vandermonde factor matrix}

In Section IV] it will become clear that existing MHR uniqueness results (e.g. [16], [24], [22], [23]) can often be explained in terms of a Vandermonde constrained CPD, defined next. Consider the PD of $\mathcal{X}=\sum_{r=1}^{R} \mathbf{a}_{r} \otimes \mathbf{b}_{r} \otimes \mathbf{c}_{r} \in$ $\mathbb{C}^{I \times J \times K}$ in (1) where $\mathbf{A}$ is Vandermonde, which can be interpreted as a 1D HR factorization with an additional diversity. The fact that $\mathbf{A}$ is Vandermonde may increase the minimal $R$ in (1). This minimal value will be denoted by $r_{\mathrm{VDM}}(\mathcal{X})$. If some of the factor matrices are Vandermonde and $R=r_{\mathrm{VDM}}(\mathcal{X})$, then (1) will be called a VDMCPD of $\mathcal{X}$. In a VDM-CPD the scaling/counterscaling indeterminacies do not involve the Vandermonde factors. If at least one of the factor matrices has full column rank, we may ignore the Vandermonde structure and establish uniqueness via the CPD theorems in Section II-A If none of the factor matrices has full column rank, we may use spatial smoothing to generate a PD in which at least one factor matrix has full column rank. In particular, using spatial smoothing and mode combination, as explained Subsection II-C, the original Vandermonde constrained PD can first be transformed into the matrix factorization $\mathbf{Z}=\sum_{r=1}^{R}\left(\mathbf{a}_{r}^{\left(K_{1}\right)} \otimes \mathbf{b}_{r}\right)\left(\mathbf{a}_{r}^{\left(L_{1}\right)} \otimes \mathbf{c}_{r}\right) \in \mathbb{C}^{K_{1} J \times L_{1} K}$ subject to $K_{1}+L_{1}=I+1$. Exploiting the shift-invariance of $\mathbf{A}^{\left(K_{1}\right)}$ a second time yields the Vandermonde constrained PD $\boldsymbol{Y}=\sum_{r=1}^{R}\left(\frac{1}{z_{r}}\right) \otimes\left(\underline{\mathbf{a}}_{r}^{\left(K_{1}\right)} \otimes \mathbf{b}_{r}\right) \otimes\left(\mathbf{a}_{r}^{\left(L_{1}\right)} \otimes \mathbf{c}_{r}\right) \in \mathbb{C}^{2 \times\left(K_{1}-1\right) J \times L_{1} K}$ with matrix representation

$\mathbf{Y}^{(n)}=\left[\begin{array}{c}\left(\underline{\mathbf{I}}_{I} \otimes \mathbf{I}_{J}\right) \mathbf{Z} \\ \left(\overline{\mathbf{I}_{I}} \otimes \mathbf{I}_{J}\right) \mathbf{Z}\end{array}\right]=\left(\mathbf{A}^{(2)} \odot\left(\underline{\mathbf{A}}^{\left(K_{1}\right)} \odot \mathbf{B}\right)\right)\left(\mathbf{A}^{\left(L_{1}\right)} \odot \mathbf{C}\right)^{T}$,

where $\mathbf{A}^{(2)}=\left[\begin{array}{ccc}1 & \cdots & 1 \\ z_{1} & \cdots & z_{R}\end{array}\right] \in \mathbb{C}^{2 \times R}$. We now have the following variant of Theorem III.3. 
Theorem III.1. Consider the PD of $\mathcal{X} \in \mathbb{C}^{I \times J \times K}$ in (1). Assume that $\boldsymbol{A}$ is a Vandermonde with generators $\left\{z_{r}\right\}$. If there exists a pair $\left(K_{1}, L_{1}\right)$ subject to $K_{1}+L_{1}=I+1$ such that

$$
\left\{\begin{array}{l}
\boldsymbol{A}^{\left(L_{1}\right)} \odot \boldsymbol{C} \text { has full column rank, } \\
\underline{A}^{\left(K_{1}\right)} \odot \boldsymbol{B} \text { has full column rank, } \\
z_{r} \neq z_{s}, \forall r \neq s,
\end{array}\right.
$$

then $R=r_{V D M}(\mathcal{X})$ and the VDM-CPD of $\mathcal{X}$ is unique. Generically ${ }^{2}$ condition (22) is satisfied if and only if $\left\lceil\frac{R}{J}\right\rceil+$ $\left\lceil\frac{R}{K}\right\rceil \leqslant I$.

Note that if $R \leqslant K$, then the generic condition in Theorem III.1 simplifies to $R \leqslant(I-1) J=\left(1-\frac{1}{I}\right) I J$. Using tools from algebraic geometry it can be verified that a generic necessary uniqueness condition is that the number of VDM-CPD parameters $(J+K) R$ does not exceed the number of tensor entries $I J K$, implying that $R \leqslant \frac{I J K}{J+K}=\left(\frac{I J}{\frac{I}{K}+1}\right) K$ must be satisfied. In other words, if $R \leqslant K$, then the generic condition $R \leqslant(I-1) J$ is both necessary and sufficient. In cases where not necessarily $R \leqslant K$, the bound in Theorem III.1 can generically be expressed as $R \leqslant \min \left(\left(K_{1}-1\right) J, L_{1} K\right)=\min ((1-$ $\left.\left.\frac{1}{K_{1}}\right) K_{1} J, L_{1} K\right)$. An algebraic algorithm for computing the CPD with a Vandermonde factor matrix was provided in [37].

\section{New uniqueness Conditions for MHR}

It was recognized in [32] that $N$-dimensional HR problems can be formulated in terms of the constrained PD of a tensor $\mathcal{X} \in \mathbb{C}^{I_{1} \times \cdots \times I_{N} \times M}$,

$$
\mathcal{X}=\sum_{r=1}^{R} \mathbf{a}_{r}^{(1)} \otimes \cdots \otimes \mathbf{a}_{r}^{(N)} \otimes \mathbf{c}_{r},
$$

with Vandermonde factor matrices $\mathbf{A}^{(n)}=$ $\left[\mathbf{a}_{1}^{(n)}, \ldots, \mathbf{a}_{R}^{(n)}\right] \in \mathbb{C}^{I_{n} \times R}$ with $\mathbf{a}_{r}^{(n)}=\left[1, z_{r, n}, z_{r, n}^{2}, \ldots, z_{r, n}^{I_{n}-1}\right]^{T}$ and unstructured $\mathbf{C}=\left[\mathbf{c}_{1}, \ldots, \mathbf{c}_{R}\right] \in \mathbb{C}^{M \times R}$, where $M$ is the number of snapshots and $R$ is the number of exponentials. In order to stress that $\mathbf{A}^{(1)}, \ldots, \mathbf{A}^{(N)}$ are all Vandermonde, the Vandermonde constrained rank of $\mathcal{X}$ will be denoted by $r_{\mathrm{MHR}}(\mathcal{X})$.

The PD of $X$ in 23) has the following matrix representation

$$
\mathbf{X}=\left(\mathbf{A}^{(1)} \odot \cdots \odot \mathbf{A}^{(N)}\right) \mathbf{C}^{T} \in \mathbb{C}^{\left(\prod_{n=1}^{N} I_{n}\right) \times M} .
$$

Note that (24) is a MHR generalization of (19). The cases where $M=1$ are referred to as single-snapshot problems while the cases where $M>1$ are referred to as multiple snapshot problems. The goal of MHR is to recover the generators $\left\{z_{r, n}\right\}$ from the observed data tensor $\mathcal{X}$.

In Subsection IV-A we first present a generic sufficient and "almost necessary" uniqueness condition for MHR, which will demonstrate that existing results do not

\footnotetext{
${ }^{2}$ A generic Vandermonde constrained (C)PD property is a property that holds with probability one if the entries of the unstructured factor matrices and the generators of the Vandermonde factor matrices are randomly drawn from continuous distributions.
}

fully exploit the MHR structure. In Subsection IV-B we will present a link between the MHR problem and the coupled CPD. This will allow us to formulate necessary and sufficient deterministic uniqueness conditions for MHR. In several signal processing applications, such as direction-of-arrival estimation, the generators are located on the unit circle $\left(\left|z_{r, n}\right|=1\right)$. Subsection IV-C briefly extends the results to this special but important case. In particular, we explain that if $\mathbf{C}$ in (24) does not have full column rank, then FBA may relax the presented MHR uniqueness conditions.

\section{A. Generic conditions for MHR uniqueness}

Results from algebraic geometry imply that a necessary condition for generic identifiability is that the total number $(N+M) R$ of MHR parameters in (23) does not exceed the number of tensor entries $\left(\prod_{n=1}^{N} I_{n}\right) M$, i.e., $(N+M) R \leqslant\left(\prod_{n=1}^{N} I_{n}\right) M \Leftrightarrow R \leqslant\left(\frac{\prod_{n=1}^{N} I_{n}}{\frac{N}{M}+1}\right) M$. Now let us assume that $C$ in 24) has full column rank, implying that $M \geqslant R$. Combination of these two inequalities results in the necessary condition $R \leqslant \prod_{n=1}^{N} I_{n}-N$, for the case $M \geqslant R$. We now present a sufficient generic condition that differs from this bound by at most one. For the derivation of the generic uniqueness condition for the MHR decomposition (24), we resort to an algebraic geometry based tool for checking generic uniqueness of structured matrix factorizations of the form $\mathbf{X}=\mathbf{M C}^{T}$, in which the entries of the matrix $\mathbf{M}$ can be parameterized by rational functions [11]. In our MHR setting, we have $\mathbf{M}=\mathbf{A}^{(1)} \odot \cdots \odot \mathbf{A}^{(N)}$, where each entry $m_{i_{1}, \ldots, i_{N}}=z_{r, 1}^{i_{1}-1} \cdots z_{r, N}^{i_{N}-1}$ is indeed a rational function of the generators (actually it is a polynomial). In situations where $\mathbf{C}$ generically has full column rank, the decomposition of $\mathbf{X}$ is generically unique if the number of rank- 1 terms is bounded by $R \leqslant \hat{N}-\hat{l}-1$ [11, Theorem $1]$, where $\hat{l}$ is an upper bound on the number of variables needed to parameterize the vector $\mathbf{a}_{r}^{(1)} \otimes \cdots \otimes \mathbf{a}_{r}^{(N)}$, and $\widehat{N}$ is a lower bound on the dimension of the vector space spanned by the vectors in the set

$$
\left\{\mathbf{a}_{1}\left(z_{1}\right) \otimes \cdots \otimes \mathbf{a}_{N}\left(z_{N}\right) \mid z_{n} \in \mathbb{C}, 1 \leqslant n \leqslant N\right\}
$$

with $\mathbf{a}_{n}\left(z_{n}\right)=\left[\begin{array}{llll}1 & z_{n} & \ldots & z_{n}^{I_{n}-1}\end{array}\right]^{T}$. Clearly, $\hat{l}=N$, i.e., $\hat{l}$ can be taken equal to the number of generators $z_{r, 1}, \ldots, z_{r, N}$. In [16. Proposition 4] an example $]^{3}$ is given that implies that the vectors in the set (25) span the entire $\left(\prod_{n=1}^{N} I_{n}\right)$ dimensional space, i.e., $\widehat{N}=\prod_{n=1}^{N} I_{n}$. To summarize, the MHR factorization (23) is generically unique if

$$
R \leqslant M \quad \text { and } \quad R \leqslant \prod_{n=1}^{N} I_{n}-N-1 .
$$

\footnotetext{
${ }^{3}$ The example is the following. Let $z_{r, n}=e^{i \cdot 2 \pi \cdot\left(\prod_{m=1}^{N-1} I_{m}\right) \frac{r-1}{R}}$ denote the generator of the $r$ th column of the Vandermonde matrix $\mathbf{A}^{(n)} \in \mathbb{C}^{I_{n} \times R}$. By letting $R=\prod_{n=1}^{N} I_{n}$, the matrix $\mathbf{A}^{(1)} \odot \cdots \odot \mathbf{A}^{(N)} \in$ $\mathbb{C}^{\left(\prod_{n=1}^{N} I_{n}\right) \times\left(\prod_{n=1}^{N} I_{n}\right)}$ is also Vandermonde with distinct generators $1, e^{i \cdot 2 \pi \frac{1}{R}}, \ldots, e^{i \cdot 2 \pi \frac{R-1}{R}}$. This implies that the vectors in the set 25 span the entire $\left(\prod_{n=1}^{N} I_{n}\right)$-dimensional space.
} 
Let us assume w.l.o.g. that $I_{1}=\max _{1 \leqslant n \leqslant N} I_{n}$. The existing MHR uniqueness results (e.g. [16], [22], [23]) yield the more restrictive condition $R \leqslant M$ and $R \leqslant(1-$ $\left.\frac{1}{I_{1}}\right) \prod_{n=1}^{N} I_{n}$. Note that this is exactly the generic version of condition (22), in which modes 2 to $N$ of the PD of $X$ have been combined into a single factor matrix $\left(\mathbf{B}=\mathbf{A}^{(2)} \odot \cdots \odot \mathbf{A}^{(N)}\right)$. We will further elaborate in Section IV-B. The gain in terms of identifiability is most noticeable in cases where $\max _{1 \leqslant n \leqslant N} I_{n}$ is small and the number of tensor entries $\prod_{n=1}^{N} I_{n}$ is large.

\section{B. Deterministic conditions for MHR uniqueness}

1) MHR uniqueness in cases where $C$ has full column rank: Let us first consider multiple snapshot MHR cases where $\mathbf{C}$ has full column rank (implying $M \geqslant R$ ). Recall that by capitalizing on the Vandermonde structure of A in (19), spatial smoothing turns a 1D HR problem into a CPD. We will do this for all $N$ dimensions of the MHR problem, overall obtaining a coupled CPD. More precisely, using $z_{n, r}^{l_{n}+k_{n}-2}=z_{n, r}^{l_{n}-1} z_{n, r}^{k_{n}-1}$, spatial smoothing in the $n$th dimension produces the tensor $\boldsymbol{Y}^{(n)} \in$

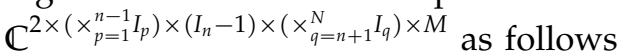

$$
\begin{aligned}
y_{k_{n}, i_{1}, \ldots, i_{n-1}, l_{n}, i_{n+1}, \ldots, i_{N}, m}^{(n)} & =x_{i_{1}, \ldots, i_{n-1}, k_{n}+l_{n}-1, i_{n+1}, \ldots, i_{N}, m} \\
& =\sum_{r=1}^{R} \prod_{p=1, p \neq n}^{N} a_{i_{p}, r}^{(p)} z_{r, n}^{k_{n}-1} z_{r, n}^{l_{n}-1} c_{m, r},
\end{aligned}
$$

where $k_{n} \in\{1,2\}$ and $l_{n} \in\left\{1, \ldots, I_{n}-1\right\}$. The PD of $\boldsymbol{y}^{(n)}$ has the following matrix representation

$$
\mathbf{Y}^{(n)}=\left(\mathbf{A}^{(2, n)} \odot \mathbf{B}^{(n)}\right) \mathbf{C}^{T},
$$

where

$$
\begin{aligned}
\mathbf{A}^{(2, n)} & =\left[\begin{array}{ccc}
1 & \cdots & 1 \\
z_{1, n} & \cdots & z_{R, n}
\end{array}\right], \\
\mathbf{B}^{(n)} & =\left(\bigodot_{p=1}^{n-1} \mathbf{A}^{(p)}\right) \odot \mathbf{A}^{\left(I_{n}-1, n\right)} \odot\left(\bigodot_{p=1}^{n-1} \mathbf{A}^{(p)}\right),
\end{aligned}
$$

in which $\mathbf{A}^{\left(I_{n}-1, n\right)}=\mathbf{A}^{(n)}\left(1: I_{n}-1,:\right)$.

Define the row-selection matrices

$$
\begin{aligned}
& \underline{\mathbf{S}}_{(n)}^{\left(I_{1}, \ldots, I_{N}\right)}=\mathbf{I}_{\prod_{p=1}^{n-1} I_{p}} \otimes \underline{\mathbf{I}}_{I_{n}} \otimes \mathbf{I}_{\prod_{q=n+1}^{N} I_{q}}, \\
& \overline{\mathbf{S}}_{(n)}^{\left(I_{1}, \ldots, I_{N}\right)}=\mathbf{I}_{\prod_{p=1}^{n-1} I_{p}} \otimes \overline{\mathbf{I}}_{I_{n}} \otimes \mathbf{I}_{\prod_{q=n+1}^{N} I_{q}},
\end{aligned}
$$

which delete the rows of $\mathbf{X}$ associated with the bottom and upper row of $\mathbf{A}^{(n)}$, respectively. In the form of $[20$, (27) can be expressed as:

$$
\mathbf{Y}^{(n)}=\left[\begin{array}{c}
\underline{\mathbf{S}}_{(n)}^{\left(I_{1}, \ldots, I_{N}\right)} \mathbf{X} \\
\overline{\mathbf{S}}_{(n)}^{\left(I_{1}, \ldots, I_{N}\right)} \mathbf{X}
\end{array}\right]=\left(\mathbf{A}^{(2, n)} \odot \mathbf{B}^{(n)}\right) \mathbf{C}^{T} .
$$

A crucial observation is that the matrix $\mathbf{C}$ does not depend on $n$. Consequently, if we consider all $n \in$ $\{1, \ldots, N\}$, then (32) represents a coupled decomposition of the form (7). Each of the individual CPDs implements the harmonic structure in the mode from which it has been derived. Summarizing, coupled CPD provides a natural framework for MHR that allows us to jointly exploit the shift-invariance structure contained in all Vandermonde matrices $\left\{\mathbf{A}^{(n)}\right\}$. In particular, the MHR factorization of $\mathcal{X}$ is unique if and only if the coupled CPD of $\left\{\boldsymbol{y}^{(1)}, \ldots, \boldsymbol{y}^{(N)}\right\}$ with factor matrices of the form (28)- 29 is unique. A necessary and sufficient condition is given in Theorem IV.1. which is an adaption of Theorem II.4 to the MHR case. It makes use of a matrix that we define for further use as

$$
\mathbf{G}^{(N)}=\left[\begin{array}{c}
C_{2}\left(\mathbf{A}^{(2,1)}\right) \odot C_{2}\left(\mathbf{B}^{(1)}\right) \\
\vdots \\
C_{2}\left(\mathbf{A}^{(2, N)}\right) \odot C_{2}\left(\mathbf{B}^{(N)}\right)
\end{array}\right] .
$$

Theorem IV.1. Consider the PD of $\mathcal{X} \in \mathbb{C}^{I_{1} \times \cdots \times I_{N} \times M}$ in (23) where the factor matrices $\left\{\boldsymbol{A}^{(n)}\right\}$ are Vandermonde. Assume that $C$ has full column rank. Then $r_{M H R}(X)=R$ and the $V D M-C P D$ of $\mathcal{X}$ is unique if and only if

$$
\boldsymbol{G}^{(N)} \boldsymbol{d}^{(2)}=\mathbf{0} \Rightarrow\|\boldsymbol{d}\|_{0} \leqslant 1,
$$

where $\boldsymbol{d}^{(2)}=\left[d_{1} d_{2}, d_{1} d_{3}, \ldots, d_{R-1} d_{R}\right]^{T} \in \mathbb{C}_{R}^{C_{R}^{2}}$.

The only possible exceptions in which (34) does not hold despite uniqueness of the VDM-CPD of $\mathcal{X}$, involve a matrix $A^{(2, n)}$ that has at least one zero entry. $\left.\right|^{4}$

Condition (34) can be hard to check in practice. On the other hand, since the bound (26) yields a sufficient generic uniqueness condition, we know that the necessary and sufficient condition (34) must be generically satisfied at least up to $R \leqslant \prod_{n=1}^{N} I_{n}-N-1$. Theorem IV.2 below provides an easy-to-check sufficient uniqueness condition that follows from Theorem II.5 At a high level, it works as follows. The conditions in Theorem IV.2 guarantee that the coupled CPD of $\left\{\boldsymbol{y}^{(1)}, \ldots, \boldsymbol{y}^{(N)}\right\}$ is unique, ignoring possible structure in the factor matrices $\left\{\mathbf{A}^{(2, n)}\right\}$ and $\left\{\mathbf{B}^{(n)}\right\}$. On the other hand, we know that $\boldsymbol{Y}^{(n)}$ can be decomposed as in (32), where $\mathbf{A}^{(2, n)}$ and $\mathbf{B}^{(n)}$ happen to have the structure in (28) and (29), respectively, $n \in\{1, \ldots, N\}$. Since there is no alternative unconstrained coupled CPD, a fortiori there is no alternative constrained coupled CPD, and hence our MHR problem has a unique solution. The "if" in Theorem IV.1 follows in the same way. The "only if" in Theorem IV.1 is more subtle. Let us assume by contradiction that Theorem IV.1 indicates that there is no uniqueness. A priori, a reason could be that there exists an alternative coupled CPD of $\left\{\boldsymbol{y}^{(1)}, \ldots, \boldsymbol{y}^{(N)}\right\}$ in which at least one of the $\mathbf{B}^{(n)}$ does not have the structure in (29). However, this possibility has been ruled out (at least if $\mathbf{A}^{(2, n)}$ is structured as in (28)) by the construction of $\boldsymbol{Y}^{(n)}$ in (32), which implements the shift-invariance. The only remaining possible cause of nonuniqueness is then that there exists an alternative coupled CPD of $\left\{\boldsymbol{y}^{(1)}, \ldots, \boldsymbol{y}^{(N)}\right\}$ in which at least one of the $\mathbf{A}^{(2, n)}$ does not have the structure in 28 . Because of

\footnotetext{
${ }^{4}$ Note that such a matrix $\mathbf{A}^{(2, n)}$ does not admit an associated VDMCPD of $\mathcal{X}$, i.e. a decomposition that involves such a matrix $\mathbf{A}^{(2, n)}$ cannot be interpreted as a solution of the MHR problem. As a result, [34] may not be satisfied while the VDM-CPD of $\mathcal{X}$ is unique.
} 
trivial CPD indeterminacies, this can be reduced to the situation in which $\mathbf{A}^{(2, n)}$ has at least one zero entry.

Theorem IV.2. Consider the PD of $\mathcal{X} \in \mathbb{C}^{I_{1} \times \cdots \times I_{N} \times M}$ in 23) where the factor matrices $\left\{\boldsymbol{A}^{(n)}\right\}$ are Vandermonde. If

$$
\left\{\begin{array}{l}
C \text { in 24) has full column rank, } \\
G^{(N)} \text { in 33) has full column rank, }
\end{array}\right.
$$

then $r_{M H R}(\mathcal{X})=R$ and the VDM-CPD of $\mathcal{X}$ is unique.

If one or more of the factor matrices $\mathbf{B}^{(1)}, \ldots, \mathbf{B}^{(N)}$ have full column rank, then MHR uniqueness can also be established via Theorem IV.3, which is an MHR adaption of Theorem $\amalg .6$.

Theorem IV.3. Consider the PD of $\mathcal{X} \in \mathbb{C}^{I_{1} \times \cdots \times I_{N} \times M}$ in (23) where the factor matrices $\left\{\boldsymbol{A}^{(n)}\right\}$ are Vandermonde. If there exists a subset $S \subseteq\{1, \ldots, N\}$ such that

$$
\left\{\begin{array}{l}
C \text { in 24) has full column rank, } \\
\boldsymbol{B}^{(n)} \text { in (29) has full column rank, } \forall n \in S, \\
\forall r \in\{1, \ldots, R\}, \forall s \in\{1, \ldots, R\} \backslash r, \exists n \in S: z_{r, n} \neq z_{s, n},
\end{array}\right.
$$

then $r_{M H R}(\mathcal{X})=R$ and the VDM-CPD of $\mathcal{X}$ is unique.

Unlike 1D HR $(N=1)$ in the case of full column rank C, for which it was explained in Section III that $\mathbf{B}^{(1)}=$ $\mathbf{A}^{\left(I_{1}-1\right)}$ having full column rank and $k_{\mathbf{A}^{(2)}} \geqslant 2 \Leftrightarrow z_{r, 1} \neq$ $z_{s, 1}, \forall r \neq s$ are necessary and sufficient, conditions $36 \mathrm{~b}$ (36c) are not necessary for MHR $(N \geqslant 2)$ in the case of full column rank $\mathbf{C}$.

2) MHR uniqueness in cases where $C$ does not have full column rank: Using spatial smoothing in a preprocessing step, Theorem IV.2 can be generalized to cases where C does not have full column rank. This is for instance relevant for single-snapshot MHR where $M=1$. By spatial smoothing of $\mathcal{X}$ in (23), we can obtain a $(2 N+1)$ thorder tensor $\mathcal{X}^{\left(K_{1}, \ldots, K_{N}\right)} \in \mathbb{C}^{K_{1} \times \cdots \times K_{N} \times L_{1} \times \cdots \times L_{N} \times M}$ as follows

$$
\begin{aligned}
x_{k_{1}, \ldots, k_{N}, l_{1}, \ldots, l_{N}, m}^{\left(K_{1}, \ldots, K_{N}\right)} & =x_{l_{1}+k_{1}-1, \ldots, l_{N}+k_{N}-1, m} \\
& =\sum_{r=1}^{R} \prod_{p=1}^{N} z_{r, p}^{k_{p}-1} \prod_{q=1}^{N} z_{r, q}^{l_{q}-1} c_{m, r},
\end{aligned}
$$

where $K_{n}+L_{n}=I_{n}+1$. Combining the first $N$ modes and the last $N+1$ modes results in the $(N+1)$ thorder tensor $\mathcal{X}_{\mathrm{SS}}^{\left(K_{1}, \ldots, K_{N}\right)} \in \mathbb{C}^{K_{1} \times \cdots \times K_{N} \times\left(\prod_{n=1}^{N} L_{n}\right) M}$ with matrix matrix representation $\mathbf{X}_{\mathrm{SS}}^{\left(K_{1}, \ldots, K_{N}\right)} \in \mathbb{C}^{\left(\prod_{n=1}^{N} K_{n}\right) \times\left(\prod_{n=1}^{N} L_{n}\right) M}$ :

$$
\mathbf{X}_{\mathrm{SS}}^{\left(K_{1}, \ldots, K_{N}\right)}=\left(\mathbf{A}^{\left(K_{1}, 1\right)} \odot \cdots \odot \mathbf{A}^{\left(K_{N}, N\right)}\right) \mathbf{C}_{\mathrm{SS}}^{T},
$$

where 'SS' stands for spatial smoothing, $\mathbf{A}^{\left(K_{n}, n\right)}=$ $\mathbf{A}^{(n)}\left(1: K_{n},:\right) \in \mathbb{C}^{K_{n} \times R}$, and

$$
\mathbf{C}_{\mathrm{SS}}=\mathbf{A}^{\left(L_{1}, 1\right)} \odot \cdots \odot \mathbf{A}^{\left(L_{N}, N\right)} \odot \mathbf{C},
$$

in which $\mathbf{A}^{\left(L_{n}, n\right)}=\mathbf{A}^{(n)}\left(1: L_{n},:\right) \in \mathbb{C}^{L_{n} \times R}$. The goal of the spatial smoothing is to obtain a matrix $\mathbf{C}_{S S}$ that has full column rank. We can now substitute $\mathbf{A}^{\left(K_{1}, 1\right)}, \ldots, \mathbf{A}^{\left(K_{N}, N\right)}$, $\mathrm{C}_{\mathrm{SS}}$ in the uniqueness conditions obtained in the previous subsections. We explicitly formulate the variants of two theorems that will be further discussed. The spatially smoothed version of (32) is given by (cf. (21)):

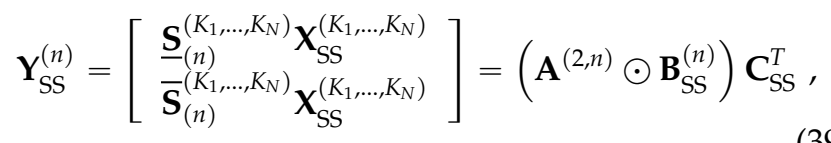

where

$$
\mathbf{B}_{\mathrm{SS}}^{(n)}=\bigodot_{p=1}^{n-1} \mathbf{A}^{\left(K_{p}, p\right)} \odot \mathbf{A}^{\left(K_{n}-1, n\right)} \odot \bigodot_{p=n+1}^{N} \mathbf{A}^{\left(K_{p}, p\right)} .
$$

In analogy with $\mathbf{G}^{(N)}$ above, matrix $\mathrm{G}_{\mathrm{SS}}^{(N)}$ is defined as

$$
\mathbf{G}_{\mathrm{SS}}^{(N)}=\left[\begin{array}{c}
C_{2}\left(\mathbf{A}^{(2,1)}\right) \odot C_{2}\left(\mathbf{B}_{\mathrm{SS}}^{(1)}\right) \\
\vdots \\
C_{2}\left(\mathbf{A}^{(2, N)}\right) \odot C_{2}\left(\mathbf{B}_{\mathrm{SS}}^{(N)}\right)
\end{array}\right] \in \mathbb{C}^{\left(\sum_{n=1}^{N} C_{J_{n}}^{2}\right) \times C_{R}^{2}} .
$$

Application of Theorem IV.2 to $\left\{\boldsymbol{y}_{\mathrm{SS}}^{(1)}, \ldots, \boldsymbol{y}_{\mathrm{SS}}^{(N)}\right\}$ yields Theorem IV.4 below, which is an extension of Theorem IV.2 to cases where $\mathbf{C}$ does not have full column rank.

Theorem IV.4. Consider the PD of $\mathcal{X} \in \mathbb{C}^{I_{1} \times \cdots \times I_{N} \times M}$ in (23) where the factor matrices $\left\{A^{(n)}\right\}$ are Vandermonde. If there exist pairs $\left\{\left(K_{n}, L_{n}\right)\right\}$ subject to $K_{n}+L_{n}=I_{n}+1$ such that

$$
\left\{\begin{array}{l}
C_{S S} \text { in } 38 \text { has full column rank, } \\
G_{S S}^{(N)} \text { in } 41 \text { has full column rank, }
\end{array}\right.
$$

then $r_{M H R}(\mathcal{X})=R$ and the VDM-CPD of $\mathcal{X}$ is unique.

Similarly, we obtain the following spatially smoothed version of Theorem IV.3 Note that the theorem also extends Theorem III.1 to the MHR case.

Theorem IV.5. Consider the PD of $\mathcal{X} \in \mathbb{C}^{I_{1} \times \cdots \times I_{N} \times M}$ in (23) where the factor matrices $\left\{A^{(n)}\right\}$ are Vandermonde. If there exists a subset $S \subseteq\{1, \ldots, N\}$ and pairs $\left\{\left(K_{n}, L_{n}\right)\right\}$ subject to $K_{n}+L_{n}=I_{n}+1$ such that

$$
\begin{aligned}
& \left\{\begin{array}{l}
C_{S S} \text { in } 38 \text { has full column rank, } \\
\boldsymbol{B}_{S S}^{(n)} \text { in } 40 \text { has full column rank, } \forall n \in S, \\
\forall r \in\{1, \ldots, R\}, \forall s \in\{1, \ldots, R\} \backslash r, \exists n \in S: z_{r, n} \neq z_{s, n},(43 \mathrm{c})
\end{array}\right. \\
& \text { then } r_{M H R}(\mathcal{X})=R \text { and the VDM-CPD of } \mathcal{X} \text { is unique. }
\end{aligned}
$$

\section{Vandermonde matrices with unit norm generators}

As explained in Section II-C, if the generators are unit-norm, then FBA can be a (partial) alternative to spatial smoothing for making sure that $\mathbf{C}$ has full column rank. In the MHR case it is required that $\left|z_{r, n}\right|=1$, $\forall r \in\{1, \ldots, R\}, \forall n \in\{1, \ldots, N\}$. FBA in all modes yields

$$
\mathbf{J}_{\prod_{n=1}^{N} I_{n}} \mathbf{X}^{*}=\left(\bigodot_{n=1}^{N} \mathbf{A}^{(n)}\right)\left(\mathbf{D}_{\mathbf{A}^{(1)}} \mathbf{D}_{\mathbf{A}^{(2)}} \cdots \mathbf{D}_{\mathbf{A}^{(N)}}\right) \mathbf{C}^{H},
$$

where $\mathbf{D}_{\mathbf{A}^{(n)}}=D_{1}\left(\left[z_{1, n}^{-\left(I_{n}-1\right)}, z_{2, n}^{-\left(I_{n}-1\right)}, \ldots, z_{R, n}^{-\left(I_{n}-1\right)}\right]\right), n \in$ $\{1, \ldots, N\}$ and $\mathbf{J}_{\prod_{n=1}^{N} I_{n}}=\bigotimes_{n=1}^{N} \mathbf{J}_{I_{n}}$. Concatenating $\mathbf{X}$ and $\mathbf{J}_{\prod_{n=1}^{N} I_{n}} \mathbf{X}^{*}$ yields

$\hat{\mathbf{X}}=\left[\mathbf{X}, \mathbf{J}_{\prod_{n=1}^{N} I_{n}} \mathbf{X}^{*}\right]=\left(\bigodot_{n=1}^{N} \mathbf{A}^{(n)}\right) \mathbf{C}_{\mathrm{FBA}}^{T} \in \mathbb{C}^{\left(\prod_{n=1}^{N} I_{n}\right) \times 2 M}$, 
where $\mathbf{C}_{\mathrm{FBA}}=\left[\mathbf{C}^{T},\left(\mathbf{D}_{\mathbf{A}^{(1)}} \mathbf{D}_{\mathbf{A}^{(2)}} \cdots \mathbf{D}_{\mathbf{A}^{(N)}}\right) \mathbf{C}^{H}\right]^{T} \in \mathbb{C}^{2 M \times R}$. We can now replace $\mathbf{C}$ by the larger matrix $\mathrm{C}_{\mathrm{FBA}}$ in all the uniqueness conditions that have been obtained.

\section{Discussion}

\section{A. Coupled CPD leads to relaxed MHR uniqueness condition}

As indicated in Section IV-A, existing uniqueness results (e.g. [16], [22], [23]) essentially derive uniqueness from the shift-invariance in a single mode and work from there to obtain an overall result. More or less explicitly, Theorem III.1 is instrumental for this. On the other hand, the joint analysis yields relaxed conditions. Here we demonstrate that Theorem IV.4 yields a more relaxed uniqueness condition than Theorem III.1 does when used for MHR. Let us first explain how MHR uniqueness can be established via Theorem III.1. Note that the factorization of each $\mathbf{Y}_{\mathrm{SS}}^{(n)}$ in 39 , and given by (39), corresponds to a matrix representation of a Vandermonde constrained CPD. Assume that condition (22) in Theorem III.1 is satisfied for $\mathbf{Y}_{\mathrm{SS}}^{(n)}$ in (39) for some $n \in\{1, \ldots, N\}$. Then $\mathbf{A}^{(2, n)}, \mathbf{B}_{\mathrm{SS}}^{(n)}$ and $\mathbf{C}_{\mathrm{SS}}$ are unique and $\mathrm{C}_{\mathrm{SS}}$ has full column rank. Since $\mathrm{C}_{\mathrm{SS}}$ has full column rank, the matrices $\left\{\mathbf{A}^{(2, n)}, \mathbf{B}_{\mathrm{SS}}^{(n)}\right\}$ can be uniquely determined via $\mathbf{Y}_{\mathrm{SS}}^{(n)}\left(\mathbf{C}_{\mathrm{SS}}^{T}\right)^{\dagger}=\mathbf{A}^{(2, n)} \odot \mathbf{B}_{\mathrm{SS}}^{(n)}, \forall n \in\{1, \ldots, N\}$, implying MHR uniqueness.

We now explain that condition (22) in Theorem III.1 implies condition (42) in Theorem IV.4 i.e., (42) is more relaxed than (22). Condition (22) implies that $z_{r, n} \neq z_{s, n}$, $\forall r \neq s$, and that $\mathbf{B}_{\mathrm{SS}}^{(n)}$ has full column rank. This is equivalent to $C_{2}\left(\mathbf{A}^{(2, n)}\right) \odot C_{2}\left(\mathbf{B}_{\mathrm{SS}}^{(n)}\right)$ having full column rank. Indeed, since the generators of $\mathbf{A}^{(2, n)}$ are assumed distinct, we know that $C_{2}\left(\mathbf{A}^{(2, n)}\right)$ is a row-vector with only non-zero entries and since $\mathbf{B}_{\mathrm{SS}}^{(n)}$ is assumed to have full column rank we also know that $C_{2}\left(\mathbf{B}_{\mathrm{SS}}^{(n)}\right)$ has full column rank [42]. This means that the submatrix $C_{2}\left(\mathbf{A}^{(2, n)}\right) \odot C_{2}\left(\mathbf{B}_{\mathrm{SS}}^{(n)}\right)$ of $\mathbf{G}_{\mathrm{SS}}^{(N)}$ has full column rank, which in turn implies that $G_{\mathrm{SS}}^{(N)}$ has full column rank. We conclude that if condition (22) in Theorem III.1 is satisfied for some $n \in\{1, \ldots, N\}$, then the condition (42) in Theorem IV.4 is automatically satisfied. However, the converse is not true. Likewise, the condition in Theorem IV.5 is more relaxed than condition (22).

Note in particular that Theorems IV.4 and IV.5 do not prevent that $k_{\mathbf{A}^{(1)}}=k_{\mathbf{A}^{(2)}}=\cdots=k_{\mathbf{A}^{(N)}}=k_{\mathbf{C}}=$ 1 , i.e., they can handle problems in which all factor matrices contain collinear columns, i.e., in every mode there are coinciding generators. On the other hand, since $C_{2}\left(\mathbf{A}^{(2, n)}\right)=\left[z_{2, n}-z_{1, n}, z_{3, n}-z_{1, n}, \ldots, z_{R, n}-z_{R-1, n}\right]$, it is also clear that condition (42) requires that for every $r \in\{1, \ldots, R\}$ there exists at least one $n \in\{1, \ldots, N\}$ and $s \in\{1, \ldots, R\}$ such that $z_{r, n} \neq z_{s, n}$. The fact that generators are not allowed to coincide in all modes is not a limitation of Theorem IV.4 but an obvious necessary requirement for MHR uniqueness.

\section{B. Efficiently checking the condition}

Let us now explain how to efficiently check the conditions in Theorem IV.4 (and in Theorem IV.2) when the dimensions $\left\{I_{1}, \ldots, I_{N}, M\right\}$ are large. Recall that $\mathbf{G}_{\mathrm{SS}}^{(N)} \in$ $\mathbb{C}^{\left(\sum_{n=1}^{N} C_{J_{n}}^{2}\right) \times C_{R}^{2}}$ in $(41)$ has full column rank if and only if $\mathbf{G}_{\mathrm{SS}}^{(N) H} \mathbf{G}_{\mathrm{SS}}^{(N)} \in \mathbb{C}_{R}^{C_{R}^{2} \times \mathrm{C}_{R}^{2}}$ is nonsingular. We will exploit the block-wise Khatri-Rao structure of $\mathrm{G}_{\mathrm{SS}}^{(N)}$ in the computation of this product. Using the properties $C_{2}(\mathbf{A})^{H}=$ $C_{2}\left(\mathbf{A}^{H}\right), C_{2}(\mathbf{A}) C_{2}(\mathbf{B})=C_{2}(\mathbf{A B})$ and $(\mathbf{A} \odot \mathbf{B})^{H}(\mathbf{A} \odot \mathbf{B})=$ $\left(\mathbf{A}^{H} \mathbf{A}\right) *\left(\mathbf{B}^{H} \mathbf{B}\right)$ we observe that

$$
\begin{aligned}
& \mathbf{G}_{\mathrm{SS}}^{(N) H} \mathbf{G}_{\mathrm{SS}}^{(N)} \\
& =\sum_{n=1}^{N}\left(C_{2}\left(\mathbf{A}^{(2, n)}\right)^{H} C_{2}\left(\mathbf{A}^{(2, n)}\right)\right) *\left(C_{2}\left(\mathbf{B}_{\mathrm{SS}}^{(n)}\right)^{H} C_{2}\left(\mathbf{B}_{\mathrm{SS}}^{(n)}\right)\right) \\
& =\sum_{n=1}^{N} D_{1}\left(C_{2}\left(\mathbf{A}^{(2, n)}\right)\right) C_{2}\left(\mathbf{B}_{\mathrm{SS}}^{(n) H} \mathbf{B}_{\mathrm{SS}}^{(n)}\right) D_{1}\left(C_{2}\left(\mathbf{A}^{(2, n) *}\right)\right),
\end{aligned}
$$

where we also exploited that $C_{2}\left(\mathbf{A}^{(2, n)}\right)$ is a row-vector. Using the identity $(\mathbf{A} \odot \mathbf{B})^{H}(\mathbf{A} \odot \mathbf{B})=\left(\mathbf{A}^{H} \mathbf{A}\right) *\left(\mathbf{B}^{H} \mathbf{B}\right)$ we also note that $\mathbf{B}_{\mathrm{SS}}^{(n) H} \mathbf{B}_{\mathrm{SS}}^{(n)}=\left(*_{m=1, m \neq n}^{N} \widetilde{\mathbf{A}}^{\left(K_{m}, m\right)}\right) * \widetilde{\mathbf{A}}^{\left(K_{n}-1, n\right)}$, in which $\widetilde{\mathbf{A}}^{(m, n)}=\mathbf{A}^{(m, n) H} \mathbf{A}^{(m, n)}$. Due to the Vandermonde structure of $\mathbf{A}^{(m, n)}$, the entries of the matrix $\mathbf{B}_{\mathrm{SS}}^{(n) H} \mathbf{B}_{\mathrm{SS}}^{(n)}$ can easily be determined as $\left(\mathbf{B}_{\mathrm{SS}}^{(n) H} \mathbf{B}_{\mathrm{SS}}^{(n)}\right)_{i j}=$ $\frac{1-\left(x_{i n}^{*} x_{j n}\right)^{K_{n}}}{1-x_{i n}^{*} x_{j n}} \prod_{m=1, m \neq n}^{N}\left(\frac{1-\left(x_{i m}^{*} x_{j m}\right)^{\left(K_{m}+1\right)}}{1-x_{i m}^{*} x_{j m}}\right)$. It is now clear that checking if $\mathbf{G}_{\mathrm{SS}}^{(N)}$ has full column rank can efficiently be done by simply checking if $\mathbf{G}_{\mathrm{SS}}^{(N) H} \mathbf{G}_{\mathrm{SS}}^{(N)}$ is nonsingular. This is possible even for large dimensions $\left\{I_{1}, \ldots, I_{N}, M\right\}$.

In cases where $R$ is very large, the construction of the $\left(C_{R}^{2} \times C_{R}^{2}\right)$ matrix $\mathbf{G}_{\mathrm{SS}}^{(N) H} \mathbf{G}_{\mathrm{SS}}^{(N)}$ can become time-consuming as well. In such cases we may instead resort to (the spatially smoothed version of) Theorem IV.3. which can easily be checked for large $R$. The spatially smoothed versions of conditions (36a) and (36b) can easily be verified by simply checking the nonsingularity of the $(R \times R)$ matrices $\mathbf{C}_{\mathrm{SS}}^{H} \mathbf{C}_{\mathrm{SS}}=\left(*_{n=1}^{N} \widetilde{\mathbf{A}}^{\left(L_{n}, n\right)}\right) *\left(\mathbf{C}^{H} \mathbf{C}\right)$ and $\mathbf{B}_{\mathrm{SS}}^{(n) H} \mathbf{B}_{\mathrm{SS}}^{(n)}=\left(*_{m=1, m \neq n}^{N} \widetilde{\mathbf{A}}^{\left(K_{m}, m\right)}\right) * \widetilde{\mathbf{A}}^{\left(K_{n}-1, n\right)}$, respectively.

Obviously Theorem IV.3 is more restrictive than Theorem IV.4 since it requires the matrices $\mathbf{B}_{\mathrm{SS}}^{(n)}$ in the spatially smoothed version of condition (36b) to have full column rank. However, Theorem IV.3 is more relaxed than Theorem III.1 (i.e., MHR uniqueness results obtained via $\mathbf{Y}^{(n)}=\left(\mathbf{A}^{(2, n)} \odot \mathbf{B}_{\mathrm{SS}}^{(n)}\right) \mathbf{C}_{\mathrm{SS}}^{T}$ for some $\left.n \in\{1, \ldots, N\}\right)$ since it does not require a mode in which all generators are distinct, as expressed by condition (36c).

\section{Generic examples}

In this section we compare i) the "almost necessary" generic MHR condition [26, possibly in combination with spatial smoothing, ii) the easy-to-check deterministic MHR condition (35) in Theorem IV.2 (and its spatially smoothed variant condition (42) in Theorem IV.4), and iii) the generic version of the 1D HR condition (22) in Theorem III.1. The examples will show that the MHR 
conditions are more relaxed than the 1D HR condition. They will also show that there is little difference between the generic implications of (26) and (35). As a first comment, note that generically $z_{r, n} \neq z_{s, n}, \forall r \neq s, \forall n \in$ $\{1, \ldots, N\}$, such that there is no interest in considering more than one value of $n$ in Theorem IV.3. In other words, generically Theorem IV.3 for MHR does not improve upon Theorem III.1 for 1D HR. Condition (22) in Theorem III.1 and condition (26) are readily available in a generic form. However, for conditions (35) and (42) in Theorems IV.2 and IV.4 no generic bounds are available. We use the following tool to check for a number of dimensionalities up to which values of $R$ conditions (35) and (42) are generically satisfied.

Lemma V.1. Let $f: \mathbb{C}^{n} \rightarrow \mathbb{C}$ be an analytic function. If there exists an element $x \in \mathbb{C}^{n}$ such that $f(\boldsymbol{x}) \neq 0$, then the set $\{\boldsymbol{x} \mid f(\boldsymbol{x})=0\}$ is of Lebesgue measure zero (e.g. [12]).

Recall that an $I \times R$ matrix has full column rank $R$ if it has a non-vanishing $R \times R$ minor. Since a minor is an analytic function (namely, it is a multivariate polynomial), Lemma V.1 can be used to verify whether the matrix generically has full column rank. The lemma implies that it suffices to numerically check condition (42) for a matrix $\mathrm{C}_{\mathrm{SS}}$ and a matrix $\mathrm{G}_{\mathrm{SS}}^{(N)}$ built from random Vandermonde matrices $\left\{\mathbf{A}^{(n)}\right\}$ and a random matrix $\mathbf{C}$.

1) $2 \mathrm{D} \mathrm{HR}$ : Let us first compare Theorem IV.2 with a result obtained in [16] for 2D HR $(N=2)$ in which $M \geqslant R$. It was explained in [16] that 2D HR uniqueness is generically guaranteed if $I_{1} I_{2}-\min \left(I_{1}, I_{2}\right) \geqslant R$ and $M \geqslant R$. This also encompasses the bounds obtained in [22], [23]. Note that this condition is the generic bound obtained from Theorem III.1. (In the context of 2D HR, the role of $(\mathbf{A}, \mathbf{B}, \mathbf{C})$ in Theorem III.1 is played by $\left(\mathbf{A}^{(1)}, \mathbf{A}^{(2)}, \mathbf{C}\right)$.)

Table II (left) indicates the maximum value of $R$ for which conditions (22), 26), (26) in combination with spatial smoothing in cases where $M=1$ and $35 / / 42$ generically guarantee uniqueness for $2 \mathrm{D}$ HR with $4 \leqslant$ $I_{1}, I_{2} \leqslant 8$ and $R \leqslant M$. In the cases listed in Table If (35) relaxes the bound on $R$ in (23) to $I_{1} I_{2}-3 \geqslant R$. Note that this generic bound is the $2 \mathrm{D}$ HR version of (26).

Table I (right) further considers single snapshot 2D HR cases $(M=1)$ in which $9 \leqslant I_{1}=I_{2} \leqslant 11$. Here the choice of spatial smoothing parameters $\left(K_{1}, K_{2}\right)$ affects the bound. Consider the example $\left(N, I_{1}, I_{2}, M\right)=$ $(2,9,9,1)$. If we choose $K_{1}=4$ and $K_{2}=6$, then condition (42) yields the upper bound $R \leqslant 21$. On the other hand, if we choose $K_{1}=K_{2}=5$, then condition (42) relaxes the upper bound to $R \leqslant 22$. The table reports the bound for the best choice of smoothing parameters.

2) $3 D$ and $4 D$ HR: Table II indicates the maximum value of $R$ for which conditions (22), 26) and (35) generically guarantee uniqueness for different $3 \mathrm{D}$ and $4 \mathrm{D}$ HR multiple snapshot configurations. The table reveals that the relaxation on $R$, obtained via the coupled CPD approach, can be quite substantial for HR problems of order greater than two.

\begin{tabular}{|c|c|c|}
\hline$M$ & 1 & 1 \\
\hline$\left(I_{1}, I_{2}, I_{3}\right)$ & $(5,5,5)$ & $(6,6,6)$ \\
\hline 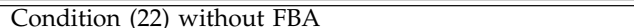 & 18 & 36 \\
\hline Condition 22) with FBA & 27 & 48 \\
\hline Condition 26) with spatial smoothing and without FBA & 27 & 40 \\
\hline Condition (26) with spatial smoothing and with FBA & 36 & 54 \\
\hline Condition 42 without FBA & 23 & 36 \\
\hline Condition 42) with FBA & 31 & 54 \\
\hline
\end{tabular}

TABLE III

Maximum VAlue for $R$ IN 23 FoR Which CONDitions 22], THE SPATIALly SMOOTHED VERSION OF 26) AND (42), WITH/WITHOUT FBA, GENERICALLY GUARANTEE UNIQUENESS FOR SINGLE SNAPSHOT 3D UNIT NORM HR.

3) Unit norm generators: Let us now compare Theorem IV.4 with results obtained in the literature for MHR with unit norm generators [22], [23]. It was explained in [22], [23] that uniqueness is generically guaranteed if $R \leqslant \min \left(\left(1-\frac{1}{K_{1}}\right) \prod_{n=1}^{N} K_{n}, 2 M \prod_{n=1}^{N} L_{n}\right)$, where w.l.o.g. it is assumed that $K_{1}=\max _{1 \leqslant n \leqslant N} K_{n}$. Compared to condition (22), the factor 2 is the result of FBA. In other words, the bound is a generic variant of Theorem III.1 for 1D HR in combination with partial smoothing and FBA. However, by simultaneously exploiting that all $\left\{\mathbf{A}^{(n)}\right\}$ are Vandermonde, improved uniqueness conditions are obtained in the coupled CPD framework.

Table III indicates the maximum value of $R$ for which conditions (22), (26) and (42) with/without FBA generically guarantee uniqueness for two different 3D HR unit norm and single-snapshot configurations. It is clear that FBA relaxes the bound on $R$.

\section{Conclusion}

MHR is a basic problem in signal processing that despite its importance, is not yet fully understood. We made a connection between MHR and coupled CPD. This led to new uniqueness conditions for MHR. We retained two values of the number of multidimensional harmonics where the transition from generically unique to generically not unique necessarily takes place. We obtained deterministic MHR uniqueness conditions that in some cases are both necessary and sufficient, implying that in some cases coupled CPD fully exploits the MHR structure. We also presented deterministic sufficient MHR conditions that are very relaxed and yet easy to check. In the companion paper [39] we explain that our approach also leads to a flexible ESPRIT-type algorithm for MHR that supports multirate sampling.

Our exposition was limited to PD with Vandermonde factor matrices. However, the results can be extended to PD with generalized Vandermonde factor matrices representing exponential polynomials [1], [5].

\section{REFERENCES}

[1] R. Badeau, B. David, and G. Richard, "High-resolution spectral analysis of mixtures of complex exponentials modulated by polynomials," IEEE Trans. Signal Process., vol. 54, no. 4, pp. 1341-1350, Apr. 2006.

[2] Y. Chen, Y. C. Eldar, and A. Goldsmith, "An algorithm for exact super-resolution and phase retrieval," in Proc. ICASSP, May 4-9, Florence, Italy, 2014. 


\begin{tabular}{|c|c|c|c|c|c|c|c|c|c|c|c|c|c|c|c|c|c|c|}
\hline$M$ & \multicolumn{5}{|c|}{$\geqslant R$} & \multicolumn{4}{|c|}{$\geqslant R$} & \multicolumn{3}{|c|}{$\geqslant R$} & \multicolumn{2}{|c|}{$\geqslant R$} & $\geqslant R$ & 1 & 1 & 1 \\
\hline$I_{\min }$ & & & 4 & & & & & & & & 6 & & & & 8 & 9 & 10 & 11 \\
\hline$I_{\max }$ & 4 & 5 & 6 & 7 & 8 & 5 & 6 & 7 & 8 & 6 & 7 & 8 & 7 & 8 & 8 & 9 & 10 & 11 \\
\hline Condition $[22$ & 12 & 16 & 20 & 24 & 28 & 20 & 25 & 30 & 35 & 30 & 36 & 42 & 42 & 49 & 56 & 20 & 25 & 30 \\
\hline Condition 26) with spatial smoothing & 13 & 17 & 21 & 25 & 29 & 22 & 27 & 32 & 37 & 33 & 39 & 45 & 46 & 53 & 61 & 22 & 27 & 33 \\
\hline Condition 35) 42 , & 13 & 17 & 21 & 25 & 29 & 22 & 27 & 32 & 37 & 33 & 39 & 45 & 46 & 53 & 61 & 22 & 27 & 33 \\
\hline
\end{tabular}

TABLE I

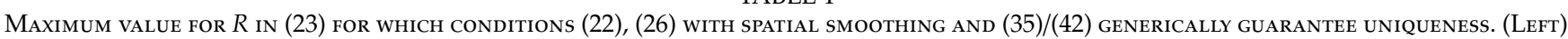
MULTIPLE SNAPSHOT 2D $\mathrm{AR}(M \geqslant R)$. (RIgHT) SINGLE SNAPSHOT 2D HR $(M=1)$. CONDITION (22) EXPLOITS HARMONIC STRUCTURE IN ONE MODE; CONDITIONS 26 AND 35/42 EXPLOIT HARMONIC STRUCTURE IN BOTH MODES. We DENOTE $I_{\min }=\min \left(I_{1}, I_{2}\right)$ AND $I_{\max }=\max \left(I_{1}, I_{2}\right)$. IN ALL CASES 35] 42 GENERICALLY REACHES THE (SPATIALLY SMOOTHED VERSION OF) BOUND 26.

\begin{tabular}{|c|c|c|c|c|c|c|c|c|c|c|c|}
\hline$M$ & \multicolumn{6}{|c|}{$\geqslant R$} & $\geqslant R$ & $\geqslant R$ & $\geqslant R$ & $\geqslant R$ & $\geqslant R$ \\
\hline$I_{4}$ & & & & & & & 2 & 3 & 3 & 3 & 3 \\
\hline$\left(I_{1}, I_{2}, I_{3}\right)$ & $(2,3,3)$ & $(3,3,3)$ & $(3,3,4)$ & $(3,4,4)$ & $(4,4,4)$ & $(4,4,5)$ & $(2,2,2)$ & $(2,2,2)$ & $(2,2,3)$ & $(2,3,3)$ & $(3,3,3)$ \\
\hline Condit & 12 & 18 & 27 & 36 & 48 & 64 & 8 & 16 & 24 & 36 & 54 \\
\hline tion (26) & 14 & 23 & 32 & 44 & 60 & 76 & 11 & 19 & 31 & 49 & 76 \\
\hline Condition (35) & 14 & 23 & 31 & 43 & 59 & 75 & 11 & 18 & 30 & 47 & 73 \\
\hline
\end{tabular}

TABLE II

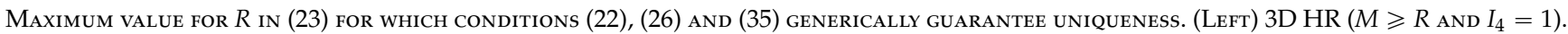

(Right) 4D HR $\left(M \geqslant R\right.$ AND $\left.I_{4}>1\right)$. CONDITION 22 EXPLOITS HARMONIC STRUCTURE IN ONE MODE; CONDITIONS 26) AND (35) EXPLOIT HARMONIC

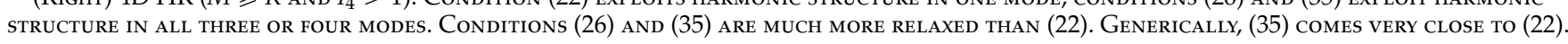

[3] L. Chiantini and G. Ottaviani, "On generic identifiability of 3tensors of small rank," SIAM J. Matrix Anal. Appl., vol. 33, no. 3, pp. 855-875, 2012.

[4] A. Cichocki, D. Mandic, C. Caifa, A.-H. Phan, G. Zhou, and L. De Lathauwer, "Tensor decompositions for signal processing applications: From two-way to multiway component analysis," IEEE Signal Processing Magazine, vol. 32, pp. 145-163, 2015.

[5] L. De Lathauwer, "Blind separation of exponential polynomials and the decomposition of a tensor in rank- $\left(L_{r}, L_{r}, 1\right)$ terms," SIAM J. Matrix Anal. Appl., vol. 32, no. 4, pp. 1451-1474, 2011.

[6] - "A link between the canonical decomposition in multilinear algebra and simultaneous matrix diagonalization," SIAM J. Matrix Anal. Appl., vol. 28, no. 3, pp. 642-666, 2006.

[7] I. Domanov and L. De Lathauwer, "On the uniqueness of the canonical polyadic decomposition of third-order tensors - Part I: Basic results and uniqueness of one factor matrix," SIAM J. Matrix Anal. Appl., vol. 34, no. 3, pp. 855-875, 2013.

[8] _ "On the uniqueness of the canonical polyadic decomposition of third-order tensors - Part II: Overall uniqueness," SIAM J. Matrix Anal. Appl., vol. 34, no. 3, pp. 876-903, 2013.

[9] - "Canonical polyadic decomposition of third-order tensors: reduction to generalized eigenvalue decomposition," SIAM J. Matrix Anal. Appl., vol. 35, no. 2, pp. 636-660, 2014.

[10] - "Generic uniqueness condition for the canonical polyadic decomposition and Indscal," SIAM J. Matrix Anal. Appl., vol. 36, no. 4, pp. 1567-1589, 2015.

[11] _ "Generic uniqueness of a structured matrix factorization and applications in blind source separation," IEEE Journal of Selected Topics in Signal Processing, vol. 10, no. 4, pp. 701-711, 2016.

[12] R. C. Gunning and H. Rossi, Analytic functions in several complex variables. Prentice-Hall, 1965.

[13] M. Haardt, F. Roemer, and G. Del Galdo, "Higher-order SVDbased subspace estimation to improve the parameter estimation accuracy in multidimensional harmonic retrieval problems," IEEE Trans. Signal Process., vol. 56, no. 7, pp. 3198-3213, Jul. 2008.

[14] R. A. Horn and C. Johnson, Matrix analysis, 2nd ed. Cambridge University Press, 2013.

[15] T. Jiang and N. D. Sidiropoulos, "Kruskal's permutation lemma and the identification of CANDECOMP/PARAFAC and bilinear model with constant modulus constraints," IEEE Trans. Signal Process., vol. 52, no. 9, pp. 2625-2636, Sep. 2004.

[16] T. Jiang, N. D. Sidiropoulos, and J. M. F. ten Berge, "Almostsure identifiability of multidimensional harmonic retrieval," IEEE Trans. Signal Process., vol. 49, no. 9, pp. 1849-1859, Sep. 2001.

[17] H. A. L. Kiers, "Towards a standardized notation and terminology in multiway analysis," J. Chemometrics, vol. 14, pp. 105-122, 2000.
[18] T. G. Kolda and B. W. Bader, "Tensor decompositions and applications," SIAM Review, vol. 51, no. 3, pp. 455-500, 2009.

[19] A. N. Lemma, A.-J. van der Veen, and E. F. Deprette, "Analysis of joint angle-frequency estimation using ESPRIT," IEEE Trans. Signal Process., vol. 51, no. 5, pp. 1264-1283, May 2003.

[20] S. E. Leurgans, R. T. Ross, and R. B. Abel, "A decomposition of three-way arrays," SIAM J. Matrix Anal. Appl., vol. 14, pp. 10641083, 1993.

[21] Y. Li, J. Razavilar, and K. J. R. Liu, "A high-resolution technique for multidimensional NMR spectroscopy," IEEE Trans. Biomed. Eng., vol. 45, no. 1, pp. 78-86, Jan. 1998.

[22] J. Liu and X. Liu, "An eigenvector-based approach for multidimensional frequency estimation with improved identifiability," IEEE Trans. Signal Process., vol. 54, no. 12, pp. 4543-4557, Dec. 2006.

[23] J. Liu, X. Liu, and W. Ma, "Multidimensional frequency estimation with finite snapshots in the presence of identical frequencies," IEEE Trans. Signal Process., vol. 55, no. 11, pp. 5179-5194, Nov. 2007.

[24] X. Liu and N. D. Sidiropoulos, "Almost sure identifiability of multidimensional constant modulus harmonic retrieval," IEEE Trans. Signal Process., vol. 50, no. 9, pp. 2366-2368, Sep. 2002.

[25] I. Maravić and M. Vetterli, "Exact sampling results for some classes of parametric nonbandlimited 2-D signals," IEEE Trans. Signal Process., vol. 52, no. 1, pp. 175-189, Jan. 2004.

[26] P. Pal and P. P. Vaidyanathan, "Nested arrays in two dimensions, Part II: Application in two dimensional array processing," IEEE Trans. Signal Processing, vol. 60, no. 9, pp. 4706-4718, Sep. 2012.

[27] A. Paulraj, R. Roy, and T. Kailath, "A subspace rotation to signal parameter estimation," Proc. of the IEEE, vol. 74, no. 7, pp. 10441045, Jul. 1986.

[28] S. U. Pillai and B. H. Kwon, "Forward/backward spatial smoothing techniques for coherent signal identification," IEEE Trans. Acoust., Speech, Signal Processing, vol. 37, no. 1, pp. 8-15, Jan. 1989.

[29] R. Roy and T. Kailath, "ESPRIT - Estimation of signal parameters via rotational invariance techniques," IEEE Trans. Acoust., Speech, Signal Processing, vol. 37, no. 7, pp. 984-995, Jul. 1989.

[30] T. J. Shan, M. Wax, and T. Kailath, "On spatial smoothing for direction-on-arrival of coherent signals," IEEE Trans. Acoust., Speech, Signal Processing, vol. 33, no. 8, pp. 806-811, Aug. 1985.

[31] B. A. Shenoy and C. S. Seelamantula, "Exact phase retrieval for a class of 2-D parametric signals," IEEE Trans. Signal Process., vol. 63, no. 1, pp. 90-103, Jan. 2015.

[32] N. D. Sidiropoulos, "Generalizing Carathéodory's uniqueness of harmonic parameterization to $N$ dimensions," IEEE Trans. Inf. Theory, vol. 47, no. 4, pp. 1687-1690, May 2001. 
[33] N. D. Sidiropoulos, R. Bro, and G. B. Giannakis, "Parallel factor analysis in sensor array processing," IEEE Trans. Signal Processing, vol. 48 , no. 8 , pp. 2377-2388, Aug. 2000.

[34] L. Sorber, M. Van Barel, and L. De Lathauwer, "Structured data fusion," IEEE Journal of Selected Topics in Signal Processing, vol. 9, no. 4, pp. 586-600, Jun. 2015.

[35] M. Sørensen and L. De Lathauwer, "Coupled tensor decompositions for applications in array signal processing," in Proc. CAMSAP, Dec. 15-18, Saint Martin, Dutch Antilles, 2013.

[36] — " "Multidimensional ESPRIT: A coupled canonical polyadic decomposition approach," in Proc. of the IEEE 8th SAM Workshop, Jun. 22-25, La Coruña, Spain, 2014.

[37] _ "Blind signal separation via tensor decompositions with a Vandermonde factor: Canonical polyadic decomposition," IEEE Trans. Signal Processing, vol. 61, no. 22, pp. 5507-5519, Nov. 2013.

[38] — "Coupled canonical polyadic decompositions and (coupled) decompositions in multilinear rank- $\left(L_{r, n}, L_{r, n}, 1\right)$ terms - Part I: Uniqueness," SIAM J. Matrix Anal. Appl., vol. 36, no. 2, pp. 496$522,2015$.

[39] , "Multidimensional harmonic retrieval via coupled canonical polyadic decompositions - Part II: Algorithm and multirate sampling," ESAT-STADIUS, KU Leuven, Belgium, Tech. Rep. 15150,2015

[40] - "New uniqueness conditions for the canonical polyadic decomposition of third-order tensors," SIAM J. Matrix Anal. Appl., vol. 36, no. 4, pp. 1381-1403, 2015.

[41] M. Sørensen, I. Domanov, and L. De Lathauwer, "Coupled canonical polyadic decompositions and (coupled) decompositions in multilinear rank- $\left(L_{r, n}, L_{r, n}, 1\right)$ terms - Part II: Algorithms," SIAM J. Matrix Anal. Appl., vol. 36, no. 3, pp. 1015-1045, 2015.

[42] A. Stegeman, "On uniqueness conditions for Candecomp/Parafac and Indscal with full column rank in one mode," Linear Algebra and Appl., vol. 431, pp. 211-227, 2009.

[43] A. Stegeman, J.M.F. ten Berge, and L. De Lathauwer, "Sufficient conditions for uniqueness in Candecomp/Parafac and Indscal with random component matrices," Psychometrika, vol. 71, pp. 219-229, 2006.

[44] V. Strassen, "Rank and optimal computation of generic tensors," Linear Algebra and Appl., vol. 52, pp. 645-685, 1983.

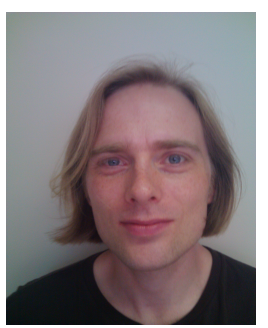

Mikael Sørensen received the Master's degree from Aalborg University, Denmark, and the Ph.D. degree from University of Nice, France, in 2006 and 2010, respectively, both in electrical engineering. Since 2010 he has been a Postdoctoral Fellow with the KU Leuven, Belgium. His research interests include applied linear algebra, tensor decompositions, and tensor-based array and signal processing.

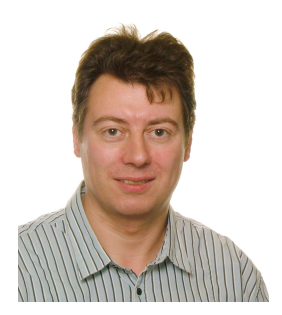

Lieven De Lathauwer received the Master's degree in electromechanical engineering and the Ph.D. degree in applied sciences from KU Leuven, Belgium, in 1992 and 1997, respectively. He is currently Professor with KU Leuven, Belgium. Dr. De Lathauwer is an Associate Editor of the SIAM Journal on Matrix Analysis and Applications and has served as an Associate Editor for the IEEE Transactions on Signal Processing. His research concerns the development of tensor tools for engineering applications. 